\title{
POSTPROCESSING THE GALERKIN METHOD: THE FINITE-ELEMENT CASE*
}

\author{
BOSCO GARCÍA-ARCHILLA ${ }^{\dagger}$ AND EDRISS S. TITI ${ }^{\ddagger}$
}

\begin{abstract}
A postprocessing technique, developed earlier for spectral methods, is extended here to Galerkin finite-element methods for dissipative evolution partial differential equations. The postprocessing amounts to solving a linear elliptic problem on a finer grid (or higher-order space) once the time integration on the coarser mesh is completed. This technique increases the convergence rate of the finite-element method to which it is applied, and this is done at almost no additional computational cost. The numerical experiments presented here show that the resulting postprocessed method is computationally more efficient than the method to which it is applied (say, quadratic finite elements) as well as standard methods of similar order of convergence as the postprocessed one (say, cubic finite elements). The error analysis of the new method is performed in $L^{2}$ and in $L^{\infty}$ norms.
\end{abstract}

Key words. dissipative equations, postprocessing finite-element methods, multilevel methods, nonlinear Galerkin methods

AMS subject classifications. 65P25, 65M70, 65M15, 65M20

\section{PII. S0036142998335893}

1. Introduction. Finite-element methods do not seem to have benefited as much as spectral methods from some of the recent advances in the dynamical systems approach to partial differential equations (PDEs) like those in [15], [14], [13], and [41]. Since these advances are mostly based on spectral decompositions, they are readily adapted to spectral methods, but do not seem to have a clear-cut translation to finite elements. In [21] and based on the results of [15] and [13], an inexpensive novel technique to increase the accuracy and computational efficiency of Fourier spectral methods was developed. In this paper, we present a general technique to improve the convergence rate of Galerkin methods which applies to finite-element methods (and in the particular case of Fourier-Galerkin methods it coincides with that in [21]; see also [37]). We do this with no direct address to [13].

Let $\Omega \subset \mathbb{R}^{d}$ be a domain with a smooth boundary. We consider dissipative PDEs (see, for instance, [8], [24], [25], [41]) which can be written as evolution systems of the form

$$
\frac{d u}{d t}+\nu A u+F(u)=0,
$$

in the Hilbert space $H$ (in this work $H=L^{2}(\Omega)$ ), with solutions determined uniquely by the initial condition

$$
u(\cdot, 0)=u_{0} .
$$

${ }^{*}$ Received by the editors March 19, 1998; accepted for publication (in revised form) March 8, 1999; published electronically January 5, 2000. This work was partially financed by DGICYT project PB95-216 and the National Science Foundation and was completed while the second author was the Orson Anderson Visiting Scholar at the Institute of Geophysics and Planetary Physics (IGPP) at the Los Alamos National Laboratory.

http://www.siam.org/journals/sinum/37-2/33589.html

${ }^{\dagger}$ Departamento de Matemáticas, Universidad Autónoma de Madrid, 28049 Madrid, Spain (bosco.garcia@usi.us.es). Current address: Depto. Matematica Aplicada II, Universidad de Sevilla, Escuela Superior de Ingenieror, Camino de lor Descubrimientor S/N, 41092 Sevilla (Spain)

${ }^{\ddagger}$ Department of Mathematics and Department of Mechanical and Aerospace Engineering, University of California, Irvine, CA 92697-3875 (etiti@math.uci.edu). 
Here, $\nu>0$ is a scalar, $A: D(A) \subset H \rightarrow H$ is a densely defined, unbounded, self-adjoint, and positive operator with compact inverse. For simplicity, we treat here only the case $A=-\Delta$, where $\Delta$ stands for the Laplacian operator subject to homogeneous Dirichlet boundary conditions, but our results also apply to other elliptic operators in divergence form with sufficiently smooth coefficients and (with adequate modifications) to other boundary conditions. The (nonlinear) operator $F$ that we consider is one of the following two types:

1. reaction-diffusion equations,

$$
F(u)=g(u),
$$

for some smooth function $g: \mathbb{R} \rightarrow \mathbb{R}$;

2. reaction-diffusion equations plus nonlinear convection,

$$
F(u)=g(u)+b(u) \cdot \nabla u,
$$

for some smooth mapping $b: \mathbb{R} \rightarrow \mathbb{R}^{d}$, and $g$ is as above.

For simplicity, we concentrate here on the two types of nonlinearity above, but both $g$ and $b$ can be made dependent on $x$ and $t$ as well. Also, although we concentrate on scalar equations, the results here can be extended to systems. We treat here the case where $\Omega \subset R^{d}$ with $d>1$; the case $d=1$ is particular and much simpler to analyze.

Let $\mathcal{T}_{h}=\left(\tau_{i}^{h}, \phi_{i}^{h}\right)_{i \in I_{h}}, h>0$, be a family of partitions of suitable domains $\Omega_{h}$, where the parameter $h$ is the maximum diameter of the elements $\tau_{i}^{h}$ in $\mathcal{T}_{h}$, and $\phi_{i}^{h}$ are the mappings of the reference simplex $\tau_{0}$ onto $\tau_{i}^{h}$. For $r \geq 2$ we consider the finite-element spaces

$$
S_{h, r}=\left\{\chi_{h} \in C\left(\bar{\Omega}_{h}\right) \mid \quad \chi_{h \mid \tau_{i}^{h}} \circ \phi_{i}^{h} \in P^{r-1}\left(\tau_{0}\right), \quad \chi_{h}(x)=0 \quad \forall x \in \partial \Omega_{h}\right\},
$$

where $P^{r-1}\left(\tau_{0}\right)$ denotes the space of polynomials of degree at most $r-1$ on $\tau_{0}$.

Let us denote by $a(\cdot, \cdot)$ the positive definite, bilinear form induced by $A$, that is, $a(u, v)=\left(A^{1 / 2} u, A^{1 / 2} u\right)=(\nabla u, \nabla v)$ for $u, v \in D\left(A^{1 / 2}\right)=H_{0}^{1}(\Omega)$, where $(\cdot, \cdot)$ denotes the standard inner product in $L^{2}(\Omega)$ (or in $\left.L^{2}(\Omega)^{d}\right)$. In a similar manner let $a_{h}(\cdot, \cdot)$ be the corresponding bilinear form on $S_{h, r}$ (in our case $a_{h}\left(\chi_{h}, \psi_{h}\right)=\left(\nabla \chi_{h}, \nabla \psi_{h}\right)_{h}$, $\chi_{h}, \psi_{h} \in S_{h, r}$, where $(\cdot, \cdot)_{h}$ stands for the standard inner product in $\left.L^{2}\left(\Omega_{h}\right)\right)$; and let us denote by $A_{h}$ the associated positive, self-adjoint operator in $S_{h, r}$, that is,

$$
a_{h}\left(\chi_{h}, \psi_{h}\right)=\left(A_{h} \chi_{h}, \psi_{h}\right)_{h}=\left(\chi_{h}, A_{h} \psi_{h}\right)_{h} \quad \forall \chi_{h}, \psi_{h} \in S_{h, r} .
$$

Let us also denote by $P_{h}$ the standard $L^{2}$ orthogonal projection onto $S_{h, r}$, and by $R_{h}$ the elliptic projection onto $S_{h, r}$ (also known as the $H^{1}$ projection) which, for $u \in H_{0}^{1}(\Omega)$ is defined by

$$
a_{h}\left(R_{h} u, \chi_{h}\right)_{h}=a_{h}\left(u, \chi_{h}\right)=\left(\nabla u, \nabla \chi_{h}\right)_{h} \quad \forall \chi_{h} \in S_{h, r} .
$$

The discrepancy between $\Omega$ and $\Omega_{h}$ is usually solved by an adequate selection of quadrature rules, although other alternatives are also possible (see section 2.1).

The Galerkin approximation to the solution $u$ of (1)-(2) is described as follows: Find $u_{h}:[0, T] \rightarrow S_{h, r}$ such that

$$
\frac{d}{d t} u_{h}+\nu A_{h} u_{h}+P_{h} F\left(u_{h}\right)=0
$$




$$
u_{h}(0)=R_{h} u_{0} .
$$

We use (6) for simplicity in the analysis. Our results may be easily extended to the case where $u_{h}(0)=P_{h} u_{0}$.

Next, we motivate and introduce the method studied here. Suppose that we are interested in the solution $u$ of (1)-(2) at a given time $T$. At time $T$, rewriting (1), we have that $\nu A u(T)=-F(u(T))-\frac{d}{d t} u(T)$. Thus, $u(T)$ can be seen as the solution of an elliptic problem whose right-hand side is not known but can be approximated. The method we propose is as follows.

(i) First, integrate (5)-(6) up to $T$ to obtain the Galerkin approximation $u_{h}(T)$.

(ii) Then solve (or, in practice, approximate) the following linear elliptic problem: Find $\tilde{u} \in D(A)$ such that

$$
\nu A \tilde{u}=-F\left(u_{h}(T)\right)-\frac{d}{d t} u_{h}(T) .
$$

We call this approximation procedure the postprocessed Galerkin method. Under certain assumptions (to be specified in section 2.1) we can state Theorem 1 below, whose proof is deferred until section 2.3 (a version of this result in $L^{\infty}$ is presented in section 4.1). In Theorem 1 and in the rest of the paper, the constants $\bar{\mu}$ and $\bar{r}$ are

$$
\bar{\mu}=\left\{\begin{array}{ll}
2 & \text { if } r \geq 4 \text { and (3) holds, } \\
1 & \text { otherwise }
\end{array} \quad \bar{r}= \begin{cases}0 & \text { if } r=3 \text { and (3) holds }, \\
1 & \text { otherwise }\end{cases}\right.
$$

Theorem 1. Let $T>0$ and $r \geq 3$. There exists a constant $C>0$ which depends on $K(u)$, defined in (22) below, such that for $h$ sufficiently small the postprocessed Galerkin approximation $\tilde{u}$ solution of (7) satisfies

$$
\|u(T)-\tilde{u}\|_{L^{2}(\Omega)}+h\|u(T)-\tilde{u}\|_{H^{1}(\Omega)} \leq C h^{r+\bar{\mu}}|\log (h)|^{\bar{r}} .
$$

We notice that the bound (8) is an improvement over the standard Galerkin error bound, $\left\|u-u_{h}\right\|_{L^{2}(\Omega)}+h\left\|u-u_{h}\right\|_{H^{1}(\Omega)} \leq C h^{r}$, which is optimal even in the case of linear problems.

We remark that in practice, of course, $\tilde{u}$ cannot be computed exactly since in general it does not belong to a finite-dimensional space, as opposed to $u_{h}$. However, one can approximate the solution $\tilde{u}$ of (7) by some $\tilde{u}_{h}$ belonging to a finite-element space of piecewise polynomials either of degree $r+\bar{\mu}-1$ over $\mathcal{T}_{h}$, or of degree $r-1$ but over some finer partition $\mathcal{T}_{h^{\prime}}$ with $h^{r+\bar{\mu}}=h^{\prime r}$.

It must be noticed that, from the practical point of view, the computational cost of the postprocessing step (7) is a very small fraction of the cost of computing $u_{h}(T)$ by time integration from $t=0$ to $t=T$. Thus, $\tilde{u}_{h}$ is an approximation to $u(T)$ of higher order (i.e., asymptotically more accurate) than $u_{h}(T)$, but it costs (almost) nothing once $u_{h}(T)$ is available. We will see the implications of this fact in the numerical experiments in section 3 .

Observe that the combination of the Galerkin method (5)-(6) and the postprocessing step (7) can be seen as a novel two-level or two-grid method for evolution equations. Two-level methods for nonlinear problems have mostly been developed and implemented for steady equations (see, e.g., [5], [31], [47], [48], and the references therein). The basic idea in the steady-state case is to solve the nonlinear problem on a low-order approximation space (or coarse grid) and then obtain a better approximation with one or two Newton iterations on a higher-order approximation space (or 
on a finer grid). In this way, the nonlinearities are treated in the (cheaper) low-order approximation space or coarse grid, and only linear systems are solved in the (more costly) higher-order approximation space.

On evolution problems, two-level (or further, multilevel) methods can obviously be used in the nonlinear systems that arise when using implicit time integrators. Other more refined ideas have recently been suggested [3], [9], [33], [34], but, so far, the two levels of discretization are used at every time step along the time evolution. On the contrary, Theorem 1 in this paper states that it is safe to perform the time evolution on the low-level (or coarse grid) approximation space and obtain the higherorder correction only once, at $t=T$, that is, when the time-marching is finished. The price to be paid is that the low-level (or coarse grid) approximation space must be composed of piecewise polynomials with a degree of at least $2(r \geq 3)$, since the postprocessing step (7) does not increase the convergence rate if $u_{h}(T)$ is computed by integrating on time on a space of piecewise-linear polynomials.

The postprocessing step (7) is a generalization to finite-element methods of an earlier postprocessing procedure developed for spectral methods in [16], [21], and [22]. To be more specific, suppose that instead of $S_{h, r}$, the approximation space is $H_{m}$ spanned by the eigenfunctions of $A$ associated with the lowest $m$ eigenvalues or frequencies. Then, the Galerkin approximation (5) would be $u_{m}:[0, T] \rightarrow H_{m}$ such that

$$
\frac{d u_{m}}{d t}+\nu A u_{m}+P_{m} F\left(u_{m}\right)=0,
$$

where $P_{m}$ is the $L^{2}$ projection onto $H_{m}$. The postprocessing step (7) would be

$$
\nu A \tilde{u}=-\frac{d u_{m}}{d t}(T)-F\left(u_{m}(T)\right) .
$$

Projecting (10) onto $H_{m}$, since $P_{m}$ and $A$ commute, we get $\nu A P_{m} \tilde{u}=-P_{m} F\left(u_{m}(T)\right)-$ $\left.\frac{d u_{m}(t)}{d t}\right|_{t=T}$, and hence, $P_{m} \tilde{u}=u_{m}(T)$, the Galerkin approximation at time $T$. Thus, one has only to compute the high-frequency modes $\tilde{q}=\left(I-P_{m}\right) \tilde{u}$. Applying $\left(I-P_{m}\right)$ to (10) we obtain

$$
\nu A \tilde{q}=-\left(I-P_{m}\right) F\left(u_{m}\right),
$$

which is the postprocessing step proposed in [21], [22]. Observe also that $\tilde{q}=\Phi\left(u_{m}\right)=$ $(\nu A)^{-1}\left(I-P_{m}\right) F\left(u_{m}\right)$.

The formulation (11) of the postprocessing step is more natural in the case of spectral methods. However, when developing this paper, (7) (or (10)) was seen to be more meaningful and useful. This point of view was also reached independently in [17], [18].

In fact, in the case of spectral methods, the postprocessed Galerkin method was developed the opposite way. In the context of inertial manifolds [14] and approximate inertial manifolds, it was shown in [13] (see also [15]) that given the low modes $P_{m} u$ of the solution $u$ of $(1)-(2), \Phi\left(P_{m} u\right)=(\nu A)^{-1}\left(I-P_{m}\right) F\left(P_{m} u\right)$ is a high-order approximation to the high-frequency modes $\left(I-P_{m}\right) u$ of $u$. Based on this fact, the so-called nonlinear Galerkin methods (NLG) [12], [28], [32] were developed (see also [10], [11], [15], [29], [42], [43], and the many references therein). In these NLG methods, nonlinear terms are typically evaluated in the whole spectrum (or some generous truncation of it), for example, $F\left(y_{m}+\Phi\left(y_{m}\right)\right)$, where $y_{m}(t) \in H_{m}$ is the low-frequency 
component of the NLG approximation $y_{m}+\Phi\left(y_{m}\right)$. This is computationally costly, and (to add to the cost) it is done along the time evolution in NLG methods.

Currently, there is enough available evidence [20], [21], [35], [46] that NLG methods are not competitive from the point of view of efficiency (or at least, not necessarily competitive). For this reason, the postprocessed Galerkin (spectral) method (9)-(10) was developed. The aim was to exploit the approximation capabilities of $\Phi$ but without paying for the computational cost of NLG methods (notice that in (9)-(10) nonlinear terms are applied only to elements of $H_{m}$, and $\Phi$ is computed only once).

The postprocessed Galerkin method was seen to be computationally more efficient than classical Galerkin spectral methods in [16] and [21]. This is also the case of Galerkin finite-element methods, as is shown in section 3 and in [30]. The computational gain is even more significant in the maximum norm. The last section of this paper is devoted to the error analysis in $L^{\infty}$.

We end this section by giving an idea of the proof of Theorem 1. Evaluate the PDE (1) at time $t=T$ and subtract it from (7). After rearranging terms and applying $A^{-1}$ one sees that

$$
u(T)-\tilde{u}=\frac{1}{\nu} A^{-1}\left(\frac{d}{d t} u_{h}(T)-\frac{d}{d t} u(T)\right)+\frac{1}{\nu} A^{-1}\left(F\left(u_{h}(T)\right)-F(u(T))\right) .
$$

Thus, at first sight, $u(T)-\tilde{u}$ depends on $u_{h}(T)-u(T)\left(\right.$ and $\left.\left(d u_{h} / d t\right)-\left.(d u / d t)\right|_{t=T}\right)$ which is only $O\left(h^{r}\right)$. However, in due course we will show that $\| A^{-1}\left(F\left(u_{h}(T)\right)-\right.$ $F(u(T))) \|_{L^{2}(\Omega)}$ can be bounded in terms of $\left\|A^{-1}\left(u(T)-u_{h}(T)\right)\right\|_{L^{2}(\Omega)}\left(A^{-1 / 2}\right.$ instead of $A^{-1}$ when $F$ is given by (4)). We write $u-u_{h}=\left(u-R_{h} u\right)+\left(R_{h} u-u_{h}\right)$. Since it is well known (see, e.g., [44]) that $\left\|A^{-1}\left(I-R_{h}\right) u(T)\right\|_{L^{2}(\Omega)}=O\left(h^{\min (2 r-2, r+2)}\right)$ (and the same bound for $\left.A^{-1}\left(I-R_{h}\right) u_{t}(T)\right)$, we have that

$$
\begin{aligned}
\|u(T)-\tilde{u}\|_{L^{2}(\Omega)} \leq & \frac{C}{\nu}\left(\left\|A^{-1}\left(u_{h}(T)-R_{h} u(T)\right)\right\|_{L^{2}(\Omega)}\right. \\
& \left.+\left\|A^{-1}\left(\frac{d}{d t} u_{h}(T)-R_{h} \frac{d}{d t} u(T)\right)\right\|_{L^{2}(\Omega)}\right)+O\left(h^{\min (2 r-2, r+2)}\right) .
\end{aligned}
$$

The $O\left(h^{\min (2 r-2, r+2)}\right)$ term above clearly shows that the postprocessed method will have a strictly higher convergence rate than the Galerkin method on which it is based, only if $r \geq 3$, that is, $u_{h}(T)$ is a piecewise polynomial with a degree of at least 2. We will duly show that the other two terms on the right-hand side of (12) are $O\left(h^{r+\bar{\mu}}|\log (h)|^{\bar{r}}\right)$.

The argument above shows that there is nothing especially nonlinear about the idea behind the postprocessed Galerkin method, and the results would be the same if $F$ were linear. Notice that even in the linear case the method presented here allows an $O\left(h^{r+2}\right)$ (or $O\left(h^{r+1}\right)$ ) error at the "same" price of a cheaper $O\left(h^{r}\right)$ method. In any case, since most of the interesting phenomena arise in nonlinear problems, for completeness we consider $F$ as in (3)-(4).

The rest of this paper is organized as follows. Section 2 is devoted to proving Theorem 1. Numerical experiments are presented in section 3. Finally, we carry the $L^{\infty}$ analysis in section 4 . 


\section{The postprocessed Galerkin method.}

2.1. Preliminaries. We now present the assumptions under which Theorem 1 holds. We restrict ourselves to quasi-uniform meshes $\mathcal{T}_{h}$, so that the following inverse estimate holds for any element $\tau \in \mathcal{T}_{h}$ and $v_{h} \in S_{h, r}$ (see, e.g., [39]):

$$
\left\|v_{h}\right\|_{W^{m, p}(\tau)} \leq C h^{l-m-d\left(\frac{1}{q}-\frac{1}{p}\right)}\left\|v_{h}\right\|_{W^{l, q}(\tau)}, \quad 0 \leq l \leq m \leq 2, \quad 1 \leq q \leq p \leq \infty .
$$

Also, we assume the following restriction between the order of approximation $r$ and the dimension $d$ :

$$
d \leq 2 r-1
$$

This is required by the presence of the nonlinear term $F$, but it can be loosened if $F$ is linear. Since finite elements are most advantageous when dealing with complex geometries, we consider only $d \geq 2$. Following the ideas presented here, the onedimensional case admits a much simpler treatment.

In this situation, for $1 \leq p \leq \infty$, and for any $v \in D(A) \cap W^{r, p}(\Omega)$, an interpolant $I_{h}(v) \in S_{h, r}$ exists such that

$$
\left\|v-I_{h}(v)\right\|_{L^{p}(\Omega)}+h\left\|v-I_{h}(v)\right\|_{W^{1, p}\left(\Omega \cap \Omega_{h}\right)} \leq C\left(h^{r}+\delta(h)\right)\|v\|_{W^{r, p}(\Omega)},
$$

(we extend by zero $I_{h}(v)$ in $\Omega \backslash \Omega_{h}$ ), where $\delta(h)=\max _{x \in \Omega_{h}} \operatorname{dist}(x, \partial \Omega)$. For $x \in \Omega_{\cap} \cap \Omega_{h}$, this bound follows from the standard theory of interpolation and the Bramble-Hilbert lemma. For $x \in \Omega \backslash \Omega_{h}, v(x)$ can be bounded using of the mean-value theorem (see, e.g., (19) below).

However, for the purpose of analysis, we may assume (see, e.g., [39]) that $\Omega_{h} \subseteq \Omega$, since one may consider a domain $\Omega_{\delta}$ with a smooth boundary such that $\Omega \cup \Omega_{h} \subset \Omega_{\delta}$, and $\max \left\{\operatorname{dist}\left(x, \partial\left(\Omega \cup \Omega_{h}\right)\right) \mid x \in \partial \Omega_{\delta}\right\} \leq c_{0} \delta(h)$ for some $c_{0}>0$. Then, for each $t$, replace $u(t)$ by the solution $u_{\delta}(t)$ of $-\Delta u_{\delta}(t)=f_{u}(t)$, where $f_{u}(t)$ is a suitable extension of $-\Delta u(t)$ to $\Omega_{\delta}$ (see [39] for details). The sets $\Omega_{\delta}$ must be built so that for $\delta$ sufficiently small we have that

$$
\left\|v_{\delta}\right\|_{W^{l+2, p}\left(\Omega_{\delta}\right)} \leq C_{p}\left\|f_{v}\right\|_{W^{l, p}\left(\Omega_{\delta}\right)}=C_{p}\|\Delta v\|_{W^{l, p}(\Omega)}, \quad 0 \leq l \leq r,
$$

for every $p \in[2, \infty)$ and every $v \in W^{r+2}(\Omega)$. Here $C_{p}>0$ constant that depends on $p$ but not on $\delta$. This can be readily done if $\Omega$ is bounded and $r+2$ smooth, or uniformly $r+2$ smooth [1, p. 67].

We will also assume that the solution of (1) satisfies

$$
M_{3}=\max _{0 \leq t \leq T}\left\|A^{3 / 2} u(\cdot, t)\right\|_{L^{2}(\Omega)}<+\infty .
$$

To ensure the estimate (16) for every solution of (3), (4), with initial data in $D\left(A^{3 / 2}\right)$, it is sufficient to require, for example, that both $g$ and $b$ satisfy $g(0)=0$ and $b(0)=0$. If, in addition, the right-hand side of (1) has a smooth forcing term $f=f(x, t)$ instead of zero, then it is sufficient that $f(x, t)$ has a zero trace at the boundary of $\Omega$.

In the understanding of the postprocessed Galerkin method, the elliptic projection $R_{h} u$ of $u$ will play a prominent role. To simplify notation we write

$$
r_{h}=R_{h} u
$$


Under these conditions the following bounds hold for $1 \leq l \leq r$ :

$$
\left.\begin{array}{r}
\left\|u-P_{h} u\right\|_{L^{2}(\Omega)} \\
\left\|u-r_{h}\right\|_{L^{2}(\Omega)}+h\left\|u-r_{h}\right\|_{H^{1}(\Omega)}
\end{array}\right\} \leq C h^{l}\|u\|_{H^{l}(\Omega)},
$$

and, for $0 \leq s \leq 2$,

$$
\left.\begin{array}{c}
\left\|A^{-s / 2}\left(u-P_{h} u\right)\right\|_{L^{2}(\Omega)} \\
\left\|A^{-s / 2}\left(u-r_{h}\right)\right\|_{L^{2}(\Omega)}
\end{array}\right\} \leq C h^{l+\bar{s}}\|u\|_{H^{l}(\Omega)}, \quad \bar{s}=\min (s, r-2) .
$$

For these bounds to hold, care must be taken to approximate the boundary with the necessary accuracy (see, e.g., [44, section 5.1]). Henceforth, we will assume that for some constant $c_{\delta}>0$,

$$
\delta(h)=\max _{x \in \Omega_{h}} \operatorname{dist}(x, \partial \Omega) \leq c_{\delta} h^{r+2} .
$$

We also remark that (16) plays a role in order to bound $\left\|\nabla\left(u-r_{h}\right)\right\|_{L^{2}(\Omega)}$ in terms of $h^{l-1}\|u\|_{H^{l}(\Omega)}$ in (17), due to the skin-layer $\Omega \backslash \Omega_{h}$. To be more precise, let $\mathcal{D} \subset \Omega$ be such that $\max \{\operatorname{dist}(x, \partial \Omega) \mid x \in \mathcal{D}\} \leq c \delta(h)$. For any $v \in D(A)$, since $v=0$ on $\partial \Omega$, one can check that

$$
\|v\|_{L^{2}(\Omega \backslash \mathcal{D})} \leq C \delta(h)\|\nabla v\|_{L^{2}(\Omega)} .
$$

Hence, taking $\mathcal{D}=\Omega_{h}$, we have that

$$
\|\nabla v\|_{L^{2}\left(\Omega \backslash \Omega_{h}\right)} \leq C\|v\|_{L^{2}\left(\Omega \backslash \Omega_{h}\right)}^{1 / 2}\|v\|_{H^{2}\left(\Omega \backslash \Omega_{h}\right)}^{1 / 2} \leq C \delta(h)^{1 / 2}\|v\|_{H^{2}(\Omega)} .
$$

For $v \in D\left(A^{3 / 2}\right)$ better estimations can be obtained as follows. Consider $\Omega_{1} \subset \subset$ $\Omega_{h}$ with a sufficiently smooth boundary such that $\max \left\{\operatorname{dist}\left(x, \partial \Omega_{h}\right) \mid x \in \Omega_{1}\right\} \leq$ $c_{0} \delta(h)$ for some $c_{0}>0$. Then $\|\nabla v\|_{L^{2}\left(\Omega \backslash \Omega_{h}\right)} \leq\|\nabla v\|_{L^{2}\left(\Omega \backslash \Omega_{1}\right)}$. Using the divergence theorem, trace theorems, and the smoothness of $\Omega$, one can show that $\|\nabla v\|_{L^{2}\left(\Omega \backslash \Omega_{1}\right)}^{2} \leq$ $\|\Delta v\|_{L^{2}\left(\Omega \backslash \Omega_{1}\right)}\left(\|v\|_{L^{2}\left(\Omega \backslash \Omega_{1}\right)}+\|\nabla v\|_{L^{2}\left(\Omega \backslash \Omega_{1}\right)}\right)$. Now, recalling (19), we have that

$$
\|\nabla v\|_{L^{2}\left(\Omega \backslash \Omega_{h}\right)} \leq C \delta(h)\|v\|_{H^{3}(\Omega)} .
$$

For the $L^{2}$ projection $P_{h}$ we will use stronger estimates than those in (17). More precisely, there exists a constant $C>0$ such that

$$
\left\|v-P_{h} v\right\|_{L^{q}(\Omega)} \leq C\|v\|_{L^{q}(\Omega)}, \quad 1 \leq q \leq \infty,
$$

$\forall v \in L^{q}(\Omega)$ (see, e.g., [44, p. 39]). Similarly, for the elliptic projection

$$
\left\|R_{h}\right\|_{\infty} \leq C
$$

(see [39]), where, here and in the rest of the paper, $\|\cdot\|_{p}$ denotes the operator norm in $\mathcal{L}\left(L^{p}(\Omega)\right.$ ) for $1 \leq p \leq \infty$. We remark that for piecewise-linear finite elements (i.e., $r=2)$ the bound $(21)$ does not hold $(C$ should be replaced by $C(1+|\log (h)|))$. In this paper, however, we restrict ourselves to $r \geq 3$.

The error estimates in sections 2.2 and 2.3, as well as the constant $C$ in Theorem 1 depend on

$$
K(u, t)=\|u(\cdot, t)\|_{H^{r}(\Omega)}+\left\|u_{t}(\cdot, t)\right\|_{H^{r}(\Omega)}, \quad K(u)=\max _{0 \leq t \leq T} K(u, t) .
$$


Notice then, that we are assuming sufficient smoothness of the solution as well as of the initial condition. It must be stated that if (1) is autonomous and $g$ and $b$ in the nonlinear terms $F$ in (3)-(4) are analytic, due to the analyticity in time of the solutions of (1)-(2), for most initial conditions $u_{0}$, it is possible to bound $\left\|u_{t}(\cdot, t)\right\|_{H^{r}(\Omega)}$ in terms of $\max _{z \in S}\|u(\cdot, z)\|_{H^{r}(\Omega)}$ for $z$ in an adequate neighborhood $S$ of $[0, T]$ in the complex plane (see, e.g., [7, pp. 104-109]).

We will frequently use the following version of Sobolev's lemma: for $p \in[1, \infty)$, there exists a constant $C=C(\Omega, p)$ such that

$$
\|v\|_{L^{p^{\prime}}(\Omega)} \leq C\|v\|_{W^{s, p}(\Omega)} \quad \text { for } \frac{1}{p} \geq \frac{1}{p^{\prime}} \geq \frac{1}{p}-\frac{s}{d} \quad \text { and } v \in W^{s, p}(\Omega)
$$

whenever $p^{\prime}<\infty$. For $p^{\prime}=\infty$ the above inequality holds for $\frac{1}{p} \geq \frac{1}{p^{\prime}}>\frac{1}{p}-\frac{s}{d}$, and, furthermore, in this case $v$ is also a continuous function.

Also the following inequality, which is easily obtained from Hölder's inequality, will be frequently used:

$$
\mid(f, v w)) \mid \leq\|f\|_{L^{2}(\Omega)}\|v\|_{L^{p}(\Omega)}\|w\|_{L^{q}(\Omega)}, \quad \frac{1}{p}+\frac{1}{q}=\frac{1}{2} .
$$

2.2. Analysis of the Galerkin method. In this section we obtain bounds for $u_{h}-r_{h}$. The main result is Theorem 2 at the end of the section. Its proof will be obtained as a consequence of several previous lemmas.

In the following, we need the residual or truncation error $T_{h}$ of the elliptic projection $r_{h}=r_{h}(t)=R_{h} u(t)$. Notice that $r_{h}$ satisfies

$$
\frac{d}{d t} r_{h}+\nu A_{h} r_{h}+P_{h} F\left(r_{h}\right)=T_{h}
$$

where the truncation error $T_{h}$ is

$$
T_{h}=P_{h}\left(F\left(r_{h}\right)-F(u)\right)+\frac{d}{d t} r_{h}-P_{h} u_{t} .
$$

In general, $\left\|T_{h}\right\|_{L^{2}(\Omega)}$ is not smaller than $\left\|u_{t}-d r_{h} / d t\right\|_{L^{2}(\Omega)}=O\left(h^{r}\right)$. In fact, the above is definitely true in the linear case when $F=0$. On the other hand, notice that in Lemma 4 below, we bound not only $\left\|T_{h}\right\|_{L^{2}(\Omega)}$ but also an integral of $e^{-\nu(t-s) A_{h}} T_{h}(s)$ with respect to $s$. It is the smoothing effect of the semigroup $e^{-\nu t A_{h}}$ that gives the extra power $h^{\bar{\mu}}|\log (h)|^{\bar{r}}$ in Theorem 2 with respect to the $O\left(h^{r}\right)$ decay of $\left\|T_{h}\right\|_{L^{2}(\Omega)}$.

LEMMA 1. Let $v_{h}:[0, T] \rightarrow S_{h, r}$ satisfy the threshold condition,

$$
\left\|r_{h}(t)-v_{h}(t)\right\|_{L^{2}(\Omega)} \leq c_{t h} h^{r},
$$

for $t \in[0, T]$. Set $\mu=0$ if the nonlinearity $F$ is given by (3); otherwise set $\mu=1$ when $F$ is given by (4). Then there exists a constant $C$, which depends on $c_{t h}$ and $\max _{0 \leq t \leq T}\|u(t)\|_{H^{r}(\Omega)}$, such that for $t \in[0, T]$

$$
\left\|A_{h}^{-\mu / 2} P_{h}\left(F\left(v_{h}(t)\right)-F\left(r_{h}(t)\right)\right)\right\|_{L^{2}(\Omega)} \leq C\left\|v_{h}(t)-r_{h}(t)\right\|_{L^{2}(\Omega)} .
$$

Furthermore, for F given by (4) we have that

$$
\begin{aligned}
\left\|P_{h}\left(F\left(v_{h}(t)\right)-F\left(r_{h}(t)\right)\right)\right\|_{L^{2}(\Omega)} \leq & C\left(\left\|\nabla\left(v_{h}(t)-r_{h}(t)\right)\right\|_{L^{2}(\Omega)}\right. \\
& \left.+h^{-1}\left\|v_{h}(t)-r_{h}(t)\right\|_{L^{2}(\Omega)}\right) .
\end{aligned}
$$


Proof. Let us take $t \in[0, T]$, and for simplicity, let us drop the explicit dependence on $t$. We first notice that both $v_{h}$ and $r_{h}$ are bounded in $L^{\infty}$ in terms of $u$, since $\left\|v_{h}\right\|_{L^{\infty}(\Omega)} \leq\left\|v_{h}-r_{h}\right\|_{L^{\infty}(\Omega)}+\left\|r_{h}\right\|_{L^{\infty}(\Omega)}$, which, thanks to the inverse estimate (13) and the threshold condition (26), is bounded by $c_{t h} h^{r-d / 2}+\left\|r_{h}\right\|_{L^{\infty}(\Omega)}$. Then in view of the fact that $R_{h}$ is bounded in $L^{\infty}$ (recall (21)), Sobolev's lemma, and the restriction (14) between the dimension $d$ and $r$ we have that $\left\|v_{h}\right\|_{L^{\infty}(\Omega)},\left\|r_{h}\right\|_{L^{\infty}(\Omega)} \leq C\|u\|_{H^{r}(\Omega)}$. As a consequence, for any smooth function $f$,

$$
f\left(v_{h}\right), f\left(r_{h}\right) \in L^{p}(\Omega) \quad \text { for } 1 \leq p \leq \infty .
$$

Thus, for $F$ as in (3), the statement (27) is a direct consequence of the mean-value theorem.

For $F$ as in (4) we first prove (27). Notice that $A_{h}^{-\mu / 2} P_{h}=A_{h}^{1-\mu / 2} A_{h}^{-1} P_{h}=$ $A_{h}^{1-\mu / 2} R_{h} A^{-1}$. In addition, for $\chi_{h} \in S_{h, r}$, we have that $\left\|A_{h}^{1 / 2} \chi_{h}\right\|_{L^{2}(\Omega)}=\left\|\nabla \chi_{h}\right\|_{L^{2}(\Omega)}$, and $\left\|A^{-1} \cdot\right\|_{H^{1}(\Omega)} \leq C\left\|A^{-1 / 2} \cdot\right\|_{L^{2}(\Omega)}$. Then, using (17) we have that

$$
\left\|A_{h}^{-1 / 2} P_{h}\left(F\left(v_{h}\right)-F\left(r_{h}\right)\right)\right\|_{L^{2}(\Omega)} \leq C\left\|A^{-1 / 2}\left(F\left(v_{h}\right)-F\left(r_{h}\right)\right)\right\|_{L^{2}(\Omega)} .
$$

Let us estimate the right-hand side above by duality. Since $A^{-1 / 2}$ is bounded, we need only to study the convection term. Let us denote $e_{h}=v_{h}-r_{h}$. We have that

$$
b\left(v_{h}\right) \cdot \nabla v_{h}-b\left(r_{h}\right) \cdot \nabla r_{h}=b\left(v_{h}\right) \cdot \nabla e_{h}+\left(b\left(v_{h}\right)-b\left(r_{h}\right)\right) \cdot \nabla r_{h} .
$$

Let $\phi \in C_{0}^{\infty}(\Omega)$ be a test function. Taking the inner product in (30) with $\phi$ and integrating by parts the first term on the right-hand side above becomes

$$
\begin{aligned}
\left(b\left(v_{h}\right) \cdot \nabla e_{h}, A^{-1 / 2} \phi\right) & =\left(e_{h}, \operatorname{div}\left(b\left(v_{h}\right) A^{-1 / 2} \phi\right)\right) \\
& \left.=\left(e_{h}, b^{\prime}\left(v_{h}\right) \cdot \nabla v_{h} A^{-1 / 2} \phi\right)+\left(e_{h}, b\left(v_{h}\right) \cdot \nabla A^{-1 / 2} \phi\right)\right) .
\end{aligned}
$$

We now take $p$ such that

$$
\frac{1}{p}=\left\{\begin{array}{cl}
1 / 4 & \text { if } d=2 \\
1 / 2-1 / d & \text { if } d>2
\end{array}\right.
$$

Then, by Sobolev's lemma, we have that $\|\cdot\|_{L^{p}(\Omega)} \leq C\|\cdot\|_{H^{1}(\Omega)}$, and, in particular, $\left\|A^{-1 / 2} \phi\right\|_{L^{p}(\Omega)} \leq C\|\phi\|_{L^{2}(\Omega)}$. We also take $q$ such that $1 / q+1 / p=1 / 2$. For this value of $q$, thanks to (14), it is immediate to check that

$$
\|u\|_{W^{1, q}(\Omega)} \leq C\|u\|_{H^{r}(\Omega)} .
$$

Applying (23) to the right-hand side of (31), we have that

$$
\begin{aligned}
\left|\left(e_{h}, b^{\prime}\left(v_{h}\right) \cdot \nabla v_{h} A^{-1 / 2} \phi\right)\right| & \leq C\left\|e_{h}\right\|_{L^{2}(\Omega)}\left\|b^{\prime}\left(v_{h}\right)\right\|_{L^{\infty}(\Omega)}\left\|\nabla v_{h}\right\|_{L^{q}(\Omega)}\|\phi\|_{L^{2}(\Omega)}, \\
\left|\left(e_{h}, b\left(v_{h}\right) \cdot \nabla A^{-1 / 2} \phi\right)\right| & \leq C\left\|e_{h}\right\|_{L^{2}(\Omega)}\left\|b\left(v_{h}\right)\right\|_{L^{\infty}(\Omega)}\|\phi\|_{L^{2}(\Omega)} .
\end{aligned}
$$

Also, for the second term on the right-hand side of (30), thanks to the mean-value theorem and (29), arguing as above we have that

$$
\left|\left(\left(b\left(v_{h}\right)-b\left(r_{h}\right)\right) \nabla r_{h}, A^{-1 / 2} \phi\right)\right| \leq C\left\|e_{h}\right\|_{L^{2}(\Omega)}\left\|\nabla r_{h}\right\|_{L^{q}(\Omega)}\|\phi\|_{L^{2}(\Omega)} .
$$

Thus, (27) is proved, provided we show that

$$
\left\|\nabla v_{h}\right\|_{L^{q}(\Omega)},\left\|\nabla r_{h}\right\|_{L^{q}(\Omega)} \leq C\|u\|_{H^{r}(\Omega)}
$$


for some $C>0$, independent of $u$. For this purpose we write

$$
\left\|\nabla v_{h}\right\|_{L^{q}(\Omega)}=\left\|\nabla\left(v_{h}-r_{h}\right)\right\|_{L^{q}(\Omega)}+\left\|\nabla r_{h}\right\|_{L^{q}(\Omega)} .
$$

On the one hand, due to the inverse estimate (13) and the threshold condition (26), we have that $\left\|\nabla\left(v_{h}-r_{h}\right)\right\|_{L^{q}(\Omega)} \leq C h^{-1+d(1 / q-1 / 2)}\left\|v_{h}-r_{h}\right\|_{L^{2}(\Omega)} \leq C c_{t h}$ (assuming $h<1$ if $d=2$ ). On the other hand, for $\nabla r_{h}$ we write $\nabla r_{h}=\nabla\left(r_{h}-I_{h}(u)\right)+\nabla I_{h}(u)$, where $I_{h}(u)$ is the interpolant of $u$. Using the inverse estimate (13), we have that

$$
\left\|\nabla r_{h}\right\|_{L^{q}(\Omega)} \leq C h^{-1+d(1 / q-1 / 2)}\left\|r_{h}-I_{h}(u)\right\|_{L^{2}(\Omega)}+\left\|\nabla I_{h}(u)\right\|_{L^{q}(\Omega)} .
$$

By writing $r_{h}-I_{h}(u)=\left(r_{h}-u\right)+\left(u-I_{h}(u)\right)$, in view of (17) and (15), it follows that $\left\|\nabla r_{h}\right\|_{L^{q}(\Omega)} \leq C\left(\|u\|_{H^{r}(\Omega)}+\|u\|_{W^{1, q}(\Omega)}\right)$. This and (32) lead to (33).

To prove (28), as before, we need only to treat the convection term, so we concentrate on (30). Therefore, because of (29) and by using the mean-value theorem, we have that

$$
\left\|F\left(v_{h}\right)-F\left(r_{h}\right)\right\|_{L^{2}(\Omega)} \leq C\left(\left\|\nabla\left(v_{h}-r_{h}\right)\right\|_{L^{2}(\Omega)}+\left\|\nabla r_{h}\right\|_{L^{\infty}(\Omega)}\left\|v_{h}-r_{h}\right\|_{L^{2}(\Omega)}\right) .
$$

Thus, (28) is proved, provided we show that $\left\|\nabla r_{h}\right\|_{L^{\infty}(\Omega)} \leq C h^{-1}\|u\|_{H^{r}(\Omega)}$. For this purpose, we take $s=1 / d$ and then, due to the inverse estimate (13), we have that $\left\|\nabla r_{h}\right\|_{L^{\infty}(\Omega)} \leq C h^{-1}\left\|r_{h}\right\|_{L^{s}(\Omega)}$. Then, the proof is easily concluded with arguments similar to those used in proving (33).

Lemma 2. Fix $c_{t h}>0$ and $T>0$. Then there exists a constant $S>0$ such that for any $C^{1}$ mapping $v_{h}:[0, T] \rightarrow S_{h, r}$ satisfying $v_{h}(0)=r_{h}(0)$ and the threshold condition (26) for $t \in\left[0, t_{1}\right]$, where $t_{1} \leq T$ the following bound holds:

$$
\max _{0 \leq t \leq t_{1}}\left\|v_{h}(t)-r_{h}(t)\right\|_{L^{2}(\Omega)} \leq S \max _{0 \leq t \leq t_{1}}\left\|\int_{0}^{t} e^{\nu(t-s) A_{h}}\left(\hat{T}_{h}(s)-T_{h}(s)\right) d s\right\|_{L^{2}(\Omega)},
$$

where

$$
\hat{T}_{h}(s)=\frac{d}{d t} v_{h}+\nu A_{h} v_{h}+P_{h} F\left(v_{h}\right)
$$

Proof. Let us call $e_{h}=r_{h}-v_{h}$ and $E_{h}=T_{h}-\hat{T}_{h}$. Subtracting (35) from (24), and taking into account that $e_{h}(0)=0$, for $t \in\left[0, t_{1}\right]$, we have that

$$
e_{h}(t)=\int_{0}^{t} e^{-\nu(t-s) A_{h}} P_{h}\left(F\left(v_{h}(s)\right)-F\left(r_{h}(s)\right)\right) d s+\int_{0}^{t} e^{-\nu(t-s) A_{h}} E_{h}(s) d s .
$$

We take $\mu=0$ if the nonlinearity $F$ is as in (3) and $\mu=1$ otherwise. By writing $e^{-\nu(t-s) A_{h}} P_{h}\left(F\left(v_{h}(s)\right)-F\left(r_{h}(s)\right)\right)=A_{h}^{\mu / 2} e^{-\nu(t-s) A_{h}}\left(A_{h}^{-\mu / 2} P_{h}\left(F\left(v_{h}(s)\right)-F\left(r_{h}(s)\right)\right)\right.$, and using Lemma 1 , from (36) we obtain that

$$
\left\|e_{h}(t)\right\|_{L^{2}(\Omega)} \leq \frac{C}{\nu^{\mu / 2}} \int_{0}^{t} \frac{\left\|e_{h}(t)\right\|_{L^{2}(\Omega)}}{(t-s)^{\mu / 2}} d s+\max _{0 \leq t \leq T}\left\|\int_{0}^{t} e^{-\nu(t-s) A_{h}} E_{h}(s) d s\right\|_{L^{2}(\Omega)}
$$

where we have taken into account that $\left\|A_{h}^{\mu / 2} e^{-\nu(t-s) A_{h}}\right\|_{2} \leq(\nu(t-s))^{-\mu / 2}$. Then a generalized Gronwall lemma (see, e.g., $[25$, p. 6]) leads to (34).

We remark that, with minor modifications, it is easy to prove that (34) also holds when $v_{h}(0) \neq r_{h}(0)$. 
Lemma 3. Let $v \in H^{r}(\Omega) \cap H_{0}^{1}(\Omega)$ and fix $c>0$. Then, there exists a constant $C=C\left(\|v\|_{H^{r}(\Omega)}, c\right)$ such that for $\mu=0,1,2$ and $\forall \chi \in H_{0}^{1}(\Omega) \cap L^{\infty}(\Omega)$ with $\|\chi\|_{L^{\infty}(\Omega)} \leq c$, we have that

$\left\|A^{-\mu / 2}(F(v)-F(\chi))\right\|_{L^{2}(\Omega)} \leq C\left(\left\|A^{(i-\mu) / 2}(v-\chi)\right\|_{L^{2}(\Omega)}+\|v-\chi\|_{L^{q}(\Omega)}\|v-\chi\|_{H^{i}(\Omega)}\right)$,

where $i=0$ when $F$ is given by (3) and $i=1$ when $F$ is given by (4). Here $q=$ $\max \left(2, d / \mu^{\prime}\right)$, where $\mu^{\prime}=\mu-1 / 2$ if $d / \mu=2$; otherwise $\mu^{\prime}=\mu$.

Proof. We concentrate on the more difficult case of nonlinear convection (4). Observe that due to the restriction (14) on the dimension $d$ and the fact that we are demanding $\|\chi\|_{L^{\infty}(\Omega)} \leq c$, then, for any smooth function $f$, both $f(v)$ and $f(\chi)$ are in $L^{p}(\Omega)$ for $1 \leq p \leq \infty$.

We estimate the left-hand side of (37) by duality. Let us then take $\phi \in C_{0}^{\infty}(\Omega)$. Let us also take $p$ such that

$$
\frac{1}{p}= \begin{cases}1 / 2-\mu / d & \text { if } d / 2>\mu, \\ 1 / 2-(\mu-1 / 2) / d & \text { if } d / 2=\mu, \\ 0 & \text { if } d / 2<\mu .\end{cases}
$$

With this choice of $p$, due to Sobolev's inequality, we always have that

$$
\left\|A^{-\mu / 2} \phi\right\|_{L^{p}(\Omega)} \leq C\|\phi\|_{L^{2}(\Omega)} .
$$

We take $q$ such that $1 / p+1 / q=1 / 2$. Notice then that this is the value of $q$ in (37). Also, with Sobolev's inequality and (14), it is easy to show that

$$
\|\cdot\|_{W^{\mu, q}(\Omega)} \leq C\|\cdot\|_{H^{r}(\Omega)}, \quad\|\cdot\|_{L^{2 q}(\Omega)} \leq C\|\cdot\|_{H^{r-1}(\Omega)} .
$$

We notice that $\left(A^{-\mu / 2}(F(v)-F(\chi)), \phi\right)=\left((F(v)-F(\chi)), A^{-\mu / 2} \phi\right)$. We treat the nonlinear convection term $b(v) \cdot \nabla v-b(\chi) \cdot \nabla \chi$ in more detail. In the following, we denote $\epsilon=v-\chi$. Observe that

$$
b(v) \cdot \nabla v-b(\chi) \cdot \nabla \chi=b(v) \cdot \nabla \epsilon+(b(v)-b(\chi)) \cdot \nabla v-(b(v)-b(\chi)) \cdot \nabla \epsilon
$$

We first bound the last term on the right-hand side above. We have that

$$
\begin{aligned}
\left|\left((b(v)-b(\chi)) \cdot \nabla \epsilon, A^{-\mu / 2} \phi\right)\right| & \leq\|b(v)-b(\chi)\|_{L^{q}(\Omega)^{d}}\|\nabla \epsilon\|_{L^{2}(\Omega)^{d}}\left\|A^{-\mu / 2} \phi\right\|_{L^{p}(\Omega)} \\
& \leq\|b(v)-b(\chi)\|_{L^{q}(\Omega)^{d}}\|\nabla \epsilon\|_{L^{2}(\Omega)^{d}}\|\phi\|_{L^{2}(\Omega)} .
\end{aligned}
$$

Since $b$ is smooth we have that $\|b(v)-b(\chi)\|_{L^{q}(\Omega)^{d}} \leq C\|\epsilon\|_{L^{q}(\Omega)}$. Thus,

$$
\left\|A^{-\mu / 2}(b(v)-b(\chi)) \cdot \nabla \epsilon\right\|_{L^{2}(\Omega)} \leq C\|\epsilon\|_{L^{q}(\Omega)}\|\epsilon\|_{H^{1}(\Omega)} .
$$

For the first term on the right-hand side of (40) we deal with the more difficult case $\mu=2$. Integrating by parts twice and observing that the boundary terms vanish, we have that $\left(b(v) \cdot \nabla \epsilon, A^{-1} \phi\right)=\left(\nabla A^{-1} \epsilon, \nabla \operatorname{div}\left(\left(A^{-1} \phi\right) b(v)\right)\right)$. Then, the following bound is easily obtained:

$$
\begin{aligned}
\left|\left(b(v) \cdot \nabla \epsilon, A^{-1} \phi\right)\right| \leq & C\left\|\nabla A^{-1} \epsilon\right\|_{L^{2}(\Omega)^{d}}\|b(v)\|_{L^{\infty}(\Omega)}\left\|A^{-1} \phi\right\|_{H^{2}(\Omega)} \\
& +\left|\left(\nabla A^{-1} \epsilon,\left(b^{\prime}(v)(\nabla v)^{T}+\operatorname{div}(b(v)) I\right) \nabla A^{-1} \phi\right)\right| \\
& +\left|\left(\nabla A^{-1} \epsilon,\left(A^{-1} \phi\right) \nabla \operatorname{div}(b(v))\right)\right| .
\end{aligned}
$$


We can bound the last term on the right-hand side of (42) as follows:

$$
\begin{aligned}
\left|\left(\nabla A^{-1} \epsilon,\left(A^{-1} \phi\right) \nabla \operatorname{div}(b(v))\right)\right| & \leq\left\|\nabla A^{-1} \epsilon\right\|_{L^{2}(\Omega)^{d}}\left\|A^{-1} \phi\right\|_{L^{p}(\Omega)}\|\nabla \operatorname{div}(b(v))\|_{L^{q}(\Omega)^{d}} \\
& \leq C\left\|\nabla A^{-1} \epsilon\right\|_{L^{2}(\Omega)^{d}}\|\nabla \operatorname{div}(b(v))\|_{L^{q}(\Omega)^{d}}\|\phi\|_{L^{2}(\Omega)} .
\end{aligned}
$$

Since $\nabla \operatorname{div}(b(v))=\left(\nabla^{2} v\right) b^{\prime}(v)+\left(b^{\prime \prime}(v) \cdot \nabla v\right) \nabla v$, using (39) we have that

$$
\|\nabla \operatorname{div}(b(v))\|_{L^{q}(\Omega)^{d}} \leq C\left(\|v\|_{W^{2, q}(\Omega)}+\|\nabla v\|_{L^{2 q}(\Omega)^{2}}^{1 / 2} \leq C\|v\|_{H^{r}(\Omega)}\right.
$$

for some $C=C\left(\|v\|_{H^{r}(\Omega)}\right)$. It follows that

$$
\left|\left(\nabla A^{-1} \epsilon,\left(A^{-1} \phi\right) \nabla \operatorname{div}(b(v))\right)\right| \leq C\left\|\nabla A^{-1} \epsilon\right\|_{L^{2}(\Omega)^{d}}\|\phi\|_{L^{2}(\Omega)} .
$$

Using similar arguments, we obtain the following estimate for the second term on the right-hand side of (42):

$$
\left|\left(\nabla A^{-1} \epsilon,\left(b^{\prime}(v)(\nabla v)^{T}+\operatorname{div}(b(v)) I\right) \nabla A^{-1} \phi\right)\right| \leq C\left\|\nabla A^{-1} \epsilon\right\|_{L^{2}(\Omega)^{d}}\|\phi\|_{L^{2}(\Omega)} .
$$

Thus, in view of (42) and (43), it follows that

$$
\left\|A^{-1}(b(v) \cdot \nabla \epsilon)\right\|_{L^{2}(\Omega)} \leq C\left\|\nabla A^{-1} \epsilon\right\|_{L^{2}(\Omega)^{d}} .
$$

Finally, the second term on the right-hand side of (40) can be treated similarly as the first one (i.e., after applying $A^{-1}$, it can be bounded by the right-hand side of (44)). This and (41) show that for $\mu=2$,

$$
\left\|A^{-\mu / 2}(b(v) \cdot \nabla v-F(\chi) \cdot \nabla \chi)\right\|_{L^{2}(\Omega)} \leq C\left(\left\|A^{(1-\mu) / 2} \epsilon\right\|_{L^{2}(\Omega)}+\|\epsilon\|_{L^{q}(\Omega)}\|\epsilon\|_{H^{1}(\Omega)}\right) .
$$

In the case where $\mu=1$, we first notice that $\left\|A^{-1 / 2} \cdot\right\|_{L^{2}(\Omega)}=\left\|\nabla A^{-1} \cdot\right\|_{L^{2}(\Omega)}$. We then return to (40) and estimate again the first two terms by duality (recall that the third term has already been dealt with). For example, for $\phi \in C_{0}^{\infty}(\Omega)^{2}$, integrating by parts twice, we obtain $\left(\nabla A^{-1}(b(v) \cdot \nabla \epsilon), \phi\right)=\left(\epsilon, \operatorname{div}\left(\left(A^{-1} \operatorname{div}(\phi)\right) b(v)\right)\right)$. It is now easy to proceed as in the case $\mu=2$. Finally, the bound (45) in the case $\mu=0$ is a direct consequence of (40).

The arguments above can be (more easily) applied to the reaction term, yielding

$$
\left\|A^{-\mu / 2}(g(v)-g(\chi))\right\|_{L^{2}(\Omega)} \leq C\left(\left\|A^{-\mu / 2}(v-\chi)\right\|_{L^{2}(\Omega)}+\|v-\chi\|_{L^{q}(\Omega)}\|v-\chi\|_{L^{2}(\Omega)}\right)
$$

for $\mu=0,1,2$. This and (45) lead to (37).

Lemma 4. There exists a constant $C$, that depends on $K(u)$ which is given in (22), such that for $t \in[0, T]$ the following bounds hold for $\mu=1,2$ :

$$
\begin{aligned}
\left\|T_{h}(t)\right\|_{L^{2}(\Omega)} & \leq C h^{r-i}, & & \\
\left\|A_{h}^{-\mu / 2} T_{h}(t)\right\|_{L^{2}(\Omega)} & \leq C h^{r+\min (\mu-i, \bar{\mu})}, & & \mu=1,2, \\
\left\|A^{-\mu / 2} T_{h}(t)\right\|_{L^{2}(\Omega)} & \leq C h^{r+\min (\mu-i, \bar{\mu})}, & & \mu=1,2,
\end{aligned}
$$

where $i=0$ when $F$ is given by (3) and $i=1$ when $F$ is given by (4).

Proof. In view of the expression of $T_{h}$ in (25), the estimate (46) is a direct consequence of the fact that $P_{h}$ is bounded, Lemma 3 (for $\mu=0$ ) and (17). 
Concerning (47)-(48), notice that, for $\mu=1,2, A_{h}^{-\mu / 2} P_{h}=A_{h}^{1-\mu / 2} R_{h} A^{-1}$. In case $\mu=1$, observe that $\left\|A_{h}^{1 / 2} R_{h} \cdot\right\|_{L^{2}(\Omega)}=\left\|\nabla R_{h} \cdot\right\|_{L^{2}(\Omega)}=\left\|A^{1 / 2} R_{h} \cdot\right\|_{L^{2}(\Omega)}$. By writing $R_{h}=I+\left(R_{h}-I\right)$, for both $\mu=1,2$, and for $v \in L^{2}(\Omega)$, we have that

$$
\left\|A_{h}^{-\mu / 2} P_{h} v\right\|_{L^{2}(\Omega)} \leq\left\|A^{1-\mu / 2} A^{-1} v\right\|_{L^{2}(\Omega)}+\left\|A^{1-\mu / 2}\left(I-R_{h}\right) A^{-1} v\right\|_{L^{2}(\Omega)} .
$$

Since by (17), $\left\|A^{1-\mu / 2}\left(I-R_{h}\right) A^{-1}\right\|_{2}=O\left(h^{\mu}\right)$ for $\mu=1,2$, then

$$
\left\|A_{h}^{-\mu / 2} P_{h} v\right\|_{L^{2}(\Omega)} \leq\left\|A^{-\mu / 2} v\right\|_{L^{2}(\Omega)}+C h^{\mu}\|v\|_{L^{2}(\Omega)} .
$$

As a result we get

$$
\begin{aligned}
\left\|A_{h}^{-\mu / 2} T_{h}\right\|_{L^{2}(\Omega)} \leq & \left\|A^{-\mu / 2}\left(F\left(r_{h}\right)-F(u)\right)\right\|_{L^{2}(\Omega)}+\left\|A^{-\mu / 2}\left(r_{h}-u\right)_{t}\right\|_{L^{2}(\Omega)} \\
& +C h^{\mu}\left(\left\|F\left(r_{h}\right)-F(u)\right\|_{L^{2}(\Omega)}+\left\|\left(r_{h}-u\right)_{t}\right\|_{L^{2}(\Omega)}\right) .
\end{aligned}
$$

To prove (48), we observe that $A^{-\mu / 2} P_{h}=A^{-\mu / 2}\left(P_{h}-I\right)+A^{-\mu / 2}$. Hence, in view of (18), $\left\|A^{-\mu / 2} T_{h}\right\|_{L^{2}(\Omega)}$ is bounded by the right-hand side of (49). Then, applying Lemma 3 and (17)-(18), we obtain

$$
\left\|A_{h}^{-\mu / 2} T_{h}\right\|_{L^{2}(\Omega)},\left\|A^{-\mu / 2} T_{h}\right\|_{L^{2}(\Omega)} \leq C\left(h^{r+\min (\mu-i, \bar{\mu})}+h^{r-i}\left\|u-r_{h}\right\|_{L^{q}(\Omega)}\right) .
$$

To finish the proof we have to show that $\left\|u-r_{h}\right\|_{L^{q}(\Omega)}=O\left(h^{\mu}\right)$. For this purpose we write

$$
\left\|u-r_{h}\right\|_{L^{q}(\Omega)} \leq\left\|u-P_{h} u\right\|_{L^{q}(\Omega)}+\left\|P_{h} u-r_{h}\right\|_{L^{q}(\Omega)} .
$$

Thanks to (13), we have that $\left\|P_{h} u-r_{h}\right\|_{L^{q}(\Omega)} \leq C h^{d(1 / q-1 / 2)}\left\|P_{h} u-r_{h}\right\|_{L^{2}(\Omega)}$. But $d(1 / q-1 / 2)=\min \left(0, \mu^{\prime}-d / 2\right)$, and due to $(14), d(1 / q-1 / 2)=\min (0, \mu-r)$. Hence,

$$
\left\|P_{h} u-r_{h}\right\|_{L^{q}(\Omega)} \leq C h^{\min (0, \mu-r)}\left\|u-r_{h}\right\|_{L^{2}(\Omega)} \leq C h^{\mu}\|u\|_{H^{r}(\Omega)} .
$$

On the other hand, replacing $v$ by $v=u-I_{h}(u)$ in (20), where $I_{h}(u)$ is the interpolant of $u$, in view of (15) and (39), we have that

$$
\left\|u-P_{h} u\right\|_{L^{q}(\Omega)} \leq C h^{\mu}\|u\|_{W^{\mu, q}(\Omega)} \leq C h^{\mu}\|u\|_{H^{r}(\Omega)} .
$$

This, together with (51) implies that

$$
\left\|u-r_{h}\right\|_{L^{q}(\Omega)} \leq C K(u) h^{\mu} .
$$

Observe that the presence of time derivatives in (49) accounts for the presence of $u_{t}$ in (22).

LEMma 5. There exists a positive constant $C=C(K(u))$ such that

$$
\max _{0 \leq t \leq T}\left\|\int_{0}^{t} e^{-\nu(t-s) A_{h}} T_{h}(s) d s\right\|_{L^{2}(\Omega)} \leq C h^{r+\bar{\mu}}|\log (h)|^{\bar{r}} .
$$

Proof. Let us take $\mu=1$ if $r=3$ and $F$ is given by (3); otherwise we take $\mu=2$. By writing $e^{-\nu(t-s) A_{h}} T_{h}=A_{h}^{\mu / 2} e^{-\nu(t-s) A_{h}}\left(A_{h}^{-\mu / 2} T_{h}\right)$, we have that

$$
\left\|\int_{0}^{t} e^{-\nu(t-s) A_{h}} T_{h}(s) d s\right\|_{L^{2}(\Omega)} \leq\left(\int_{0}^{T}\left\|A_{h}^{\frac{\mu}{2}} e^{-\nu(t-s) A_{h}}\right\|_{2} d s\right) \max _{0 \leq t \leq T}\left\|A_{h}^{\frac{-\mu}{2}} T_{h}(t)\right\|_{L^{2}(\Omega)} .
$$


Let $\lambda_{m}, \lambda_{M}$ be the smallest and largest eigenvalues of $A_{h}$, respectively. In the case $\mu=2$ one can easily check that

$$
\left\|A_{h} e^{-\nu(t-s) A_{h}}\right\|_{2} \leq \begin{cases}\lambda_{M} e^{-\nu \lambda_{M}(t-s)} & \text { if } \nu(t-s) \leq \lambda_{M}^{-1}, \\ (\nu(t-s))^{-1} & \text { if } \lambda_{M}^{-1} \leq \nu(t-s) \leq \lambda_{m}^{-1}, \\ \lambda_{m} e^{-\nu \lambda_{m}(t-s)} & \text { if } \nu(t-s) \geq \lambda_{m}^{-1} .\end{cases}
$$

On the other hand, for the case $\mu=1$, one has $\left\|A_{h}^{1 / 2} e^{-\nu(t-s) A_{h}}\right\|_{2} \leq(\nu(t-s))^{-1 / 2}$. Taking into account that $\lambda_{m}$ is bounded from below by the coercivity constant of $a(\cdot, \cdot)=\left(A^{1 / 2}, A^{1 / 2}\right)$, and that $\lambda_{M} \leq\left\|A_{h}\right\|_{2} \leq C / h^{2}$, it follows from the above that for some constant $0<C=C(T)$,

$$
\max _{0 \leq t \leq T}\left\|\int_{0}^{t} e^{-\nu(t-s) A_{h}} T_{h}(s) d s\right\|_{L^{2}(\Omega)} \leq \frac{C}{\nu^{\mu / 2}}|\log (h)|^{\bar{r}} \max _{0 \leq t \leq T}\left\|A_{h}^{-\mu / 2} T_{h}(t)\right\|_{L^{2}(\Omega)} .
$$

Thus, (53) follows from the above and Lemma 4.

THEOREM 2. There exist positive constants $C=C(K(u))$ and $h_{0}$ given by (55) below, such that for every $h \in\left(0, h_{0}\right]$,

$$
\max _{0 \leq t \leq T}\left\|r_{h}(t)-u_{h}(t)\right\|_{L^{2}(\Omega)} \leq C h^{r+\bar{\mu}}|\log (h)|^{\bar{r}} .
$$

Proof. This theorem is a direct consequence of Lemma 2 with $v_{h}$ replaced by $u_{h}$ (and hence $\hat{T}_{h}=0$ ) and Lemma 5. It remains only to check that $v_{h}(t)=u_{h}(t)$ satisfies the threshold condition (26) for $t \in[0, T]$. This can be done by a standard continuity argument as follows. Let us take

$$
h_{0}=\min \left(\frac{c_{t h}^{2}}{(2 S C)^{2}}, e^{-2}\right),
$$

where $S$ is the stability constant in (34), $C$ is the error constant in (53), and $c_{t h}$ is the threshold constant in (26). Observe that $h_{0} \leq e^{-2}$ implies that $h^{1 / 2}|\log (h)| \leq$ $2 e^{-1}<1$, for $h \leq h_{0}$. Let us define

$$
t_{1}=t_{1}(h)=\max \left\{s \quad \mid \quad v_{h}(t)=u_{h}(t) \text { satisfies }(26) \text { for } t \in[0, s]\right\} .
$$

Since $r_{h}(0)-u_{h}(0)=0$, by continuity we have that $t_{1}>0$. We now show that $t_{1}(h) \geq T$ for $h \leq h_{0}$. Suppose that $t_{1}(h)<T$ for some $h \leq h_{0}$. Since $v_{h}=u_{h}$ satisfies $(26)$ for $t \in\left[0, t_{1}(h)\right]$, it follows that

$$
\begin{aligned}
\left\|r_{h}\left(t_{1}\right)-u_{h}\left(t_{1}\right)\right\|_{L^{2}(\Omega)} & \leq S C h^{r+\bar{\mu}}|\log (h)|^{\bar{r}} \leq S C h^{r+1 / 2}\left(h^{1 / 2}|\log (h)|\right) \\
& \leq S C h^{r+1 / 2}=h^{r} S C h^{1 / 2} \leq h^{r} S C \frac{c_{t h}}{2 S C} \leq \frac{c_{t h}}{2} h^{r} .
\end{aligned}
$$

Then, there exists $t_{2}>t_{1}$ such that (26) holds for $v_{h}=u_{h}$ and $t \in\left[0, t_{2}\right]$. But this contradicts the definition of $t_{1}$. Therefore, it must be $t_{1}(h) \geq T$.

2.3. The postprocessing step. In this section we prove Theorem 1 , but first we analyze how $\tilde{u}$ (and hence $u$ ), which lives in the infinite-dimensional space $D\left(A^{3 / 2}\right)$ (recall (16)), can be approximated in some finite-element space with an $O\left(h^{r+\bar{\mu}}|\log (h)|^{\bar{r}}\right)$ error.

We consider a finite-element space $\tilde{S}_{h}$; this will be either

(1) $\tilde{S}_{h}=S_{h^{\prime}, r}$, with $\left(h^{\prime}\right)^{r} \leq C h^{r+\bar{\mu}}|\log (r)|^{\bar{r}}$, 
that is, piecewise polynomials of degree $r$ over a finer grid; or, if $u(T) \in H^{r+\bar{\mu}}$,

(2) $\tilde{S}_{h}=S_{h, r+\bar{\mu}}$,

that is, piecewise polynomials of degree $r+\bar{\mu}$ over the same grid.

Let $\tilde{u}_{h} \in \tilde{S}_{h}$ be the solution of

$$
\nu \tilde{A}_{h} \tilde{u}_{h}=-\tilde{P}_{h}\left(F\left(u_{h}(T)\right)+\frac{d}{d t} u_{h}(T)\right),
$$

where $\tilde{P}_{h}$ is the $L^{2}$ projection onto $\tilde{S}_{h}$, and $\tilde{A}_{h}$ is the linear, positive, self-adjoint operator induced by the bilinear form $a$ when restricted to $\tilde{S}_{h}$. Observe then that $\tilde{u}_{h}$ is the elliptic projection $\tilde{R}_{h} \tilde{u}$ of $\tilde{u}$ onto $\tilde{S}_{h}$. Notice also that the right-hand side of (7) is in $H^{1}(\Omega)$ but not in $H^{2}(\Omega)$. Thus, at first sight, applying (17) we have that $\left\|\tilde{u}-\tilde{u}_{h}\right\|_{L^{2}(\Omega)}=O\left(h^{3}\right)$. However, as we will show next, $\left\|\tilde{u}-\tilde{u}_{h}\right\|_{L^{2}(\Omega)}$ is indeed of the order of $O\left(h^{r+\bar{\mu}}|\log (h)|^{\bar{r}}\right)$.

Since $\tilde{u}_{h}$ is the elliptic projection of $\tilde{u}$, it is a well-known fact that

$$
\left\|\nabla\left(\tilde{u}-\tilde{u}_{h}\right)\right\|_{L^{2}(\Omega)^{d}} \leq\left\|\nabla\left(\tilde{u}-\tilde{\chi}_{h}\right)\right\|_{L^{2}(\Omega)^{d}} \quad \forall \tilde{\chi}_{h} \in \tilde{S}_{h} .
$$

Now, take $\chi_{h}=\tilde{I}_{h}(u)$, the interpolant of $u$ in $\tilde{S}_{h}$, and write $\tilde{u}-\tilde{I}_{h}(u)=(\tilde{u}-u)+$ $\left(u-\tilde{I}_{h}(u)\right)$. Then Theorem 1 and the interpolation error (15) show that

$$
\left\|\nabla\left(\tilde{u}-\tilde{u}_{h}\right)\right\|_{L^{2}(\Omega)^{d}}=O\left(h^{r+\bar{\mu}-1}|\log (h)|^{\bar{r}}\right),
$$

which by (17) leads to $\left\|\tilde{u}-\tilde{u}_{h}\right\|_{L^{2}(\Omega)}=O\left(h^{r+\bar{\mu}}|\log (h)|^{\bar{r}}\right)$ by using standard duality arguments and (17).

Lemma 6. There exists a positive constant $C$ that depends on $K(u)$, such that the following bounds hold for $\mu=1,2$ :

$$
\begin{aligned}
\left\|A^{-1}\left(r_{h}(T)-u_{h}(T)\right)_{t}\right\|_{L^{2}(\Omega)} & \leq C\left(\left\|r_{h}(T)-u_{h}(T)\right\|_{L^{2}(\Omega)}+h^{r+\bar{\mu}}\right), \\
\left\|A^{-1 / 2}\left(r_{h}(T)-u_{h}(T)\right)_{t}\right\|_{L^{2}(\Omega)} & \leq C\left(h^{-1}\left\|r_{h}(T)-u_{h}(T)\right\|_{L^{2}(\Omega)}+h^{r+1-i}\right),
\end{aligned}
$$

where $i=0$ when $F$ is given by (3); otherwise $i=1$ when $F$ is given by (4).

Proof. We drop the explicit dependence on the argument $T$. We have that

$$
\left(r_{h}-u_{h}\right)_{t}=-\nu A_{h}\left(r_{h}-u_{h}\right)+P_{h}\left(F\left(u_{h}\right)-F\left(r_{h}\right)\right)+T_{h} .
$$

Consider the third term on the right-hand side above. We have already established bounds for $A^{-\mu / 2} T_{h}$ in Lemma 4 , for $\mu=1,2$.

For the first term on the right-hand side of (57) we write $A^{-\mu / 2} A_{h}=\left(A^{-\mu / 2}-\right.$ $\left.A_{h}^{-\mu / 2} P_{h}\right) A_{h}+A_{h}^{1-\mu / 2}$. Then, due to the inverse estimate (13), it follows that $\left\|A_{h}^{s / 2}\right\|_{2} \leq C h^{-s}$ for $s=1,2$. This and (17) lead to

$$
\left\|A^{-\mu / 2} A_{h}\left(u_{h}-r_{h}\right)\right\|_{L^{2}(\Omega)} \leq C h^{\mu-2}\left\|u_{h}-r_{h}\right\|_{L^{2}(\Omega)}, \quad \mu=1,2 .
$$

Finally, for the second term on the right-hand side of (57), by writing $A^{-\mu / 2}=$ $\left(A^{-\mu / 2}-A_{h}^{-\mu / 2}\right)+A_{h}^{-\mu / 2}$, from (17) and Lemma 1, it follows that

$$
\left\|A^{-\mu / 2} P_{h}\left(F\left(u_{h}\right)-F\left(r_{h}\right)\right)\right\|_{L^{2}(\Omega)} \leq C\left\|u_{h}-r_{h}\right\|_{L^{2}(\Omega)} .
$$

This together with (57), (58), and Lemma 4 finishes the proof. 
Proof of Theorem 1. Subtracting (7) from (1) and applying $\nu A^{-1}$ we have that

(59) $u(T)-\tilde{u}=\nu^{-1}\left(A^{-1}\left(F\left(u_{h}(T)\right)-F(u(T))\right)+A^{-1}\left(\frac{d u_{h}}{d t}(T)-\frac{d u}{d t}(T)\right)\right)$.

Since $\|\nabla \cdot\|_{L^{2}(\Omega)}=\left\|A^{1 / 2} \cdot\right\|_{L^{2}(\Omega)}$, then by Lemma 3 and an argument similar to that leading from (50) to (47), we obtain the following bounds:

$$
\begin{aligned}
& \|u-\tilde{u}\|_{L^{2}(\Omega)} \leq C\left\|A^{-1}\left(u_{h}-u\right)\right\|_{L^{2}(\Omega)}+\left\|A^{-1}\left(u_{h}-u\right)_{t}\right\|_{L^{2}(\Omega)}+\epsilon_{2}, \\
& \|u-\tilde{u}\|_{H^{1}(\Omega)} \leq C\left\|A^{-1 / 2}\left(u_{h}-u\right)\right\|_{L^{2}(\Omega)}+\left\|A^{-1 / 2}\left(u_{h}-u\right)_{t}\right\|_{L^{2}(\Omega)}+\epsilon_{1},
\end{aligned}
$$

where for $\mu=1,2, \epsilon_{\mu}=\left\|u-u_{h}\right\|_{L^{q}(\Omega)}\left\|u-u_{h}\right\|_{H^{i}(\Omega)}$, with $q$ as indicated in Lemma 3 and $i=1$ when $F$ is given by (4); otherwise $i=0$.

Let us first find an upper bound for $\epsilon_{\mu}$. We write

$$
u-r_{h}=\left(u-r_{h}\right)+\left(r_{h}-u_{h}\right) .
$$

For $r_{h}-u_{h}$, using the inverse estimate (13) and Theorem 2, we have that

$$
\left\|r_{h}-u_{h}\right\|_{L^{q}(\Omega)} \leq C h^{\frac{d}{q}-\frac{d}{2}}\left\|r_{h}-u_{h}\right\|_{L^{2}(\Omega)} \leq C K(u) h^{r+\bar{\mu}+\frac{d}{q}-\frac{d}{2}}|\log (h)|^{\bar{r}} .
$$

But $d / q-d / 2=-d / p$, which, in view of (38), allows us to write $r+\bar{\mu}-d / p \geq \bar{\mu}+\mu-1$, where we have used that $r \geq 3$ in the case $d / 2<\mu$. This and (52) lead to

$$
\left\|u-u_{h}\right\|_{L^{q}(\Omega)} \leq C K(u) h^{\min (\bar{\mu}+\mu-1, \mu)}|\log (h)|^{\bar{r}} .
$$

Using again (62), applying (17), (13), and Theorem 2, one can show that

$$
\left\|u-u_{h}\right\|_{H^{i}(\Omega)} \leq C K(u) h^{r-i},
$$

where $i=1$ when $F$ is given by (4); otherwise $i=0$. Hence, it follows that

$$
\epsilon_{2} \leq C K(u) h^{r+\bar{\mu}}|\log (h)|^{\bar{r}}, \quad \epsilon_{1} \leq C K(u) h^{r+1-i}|\log (h)|^{\bar{r}} .
$$

Let us now turn to the other terms in (60)-(61). Using (62) and taking into account that $A^{-\mu / 2}$ is bounded, we have that

$$
\begin{aligned}
\left\|A^{-\mu / 2}\left(u-u_{h}\right)\right\|_{L^{2}(\Omega)} & \leq\left\|A^{-\mu / 2}\left(u-r_{h}\right)\right\|_{L^{2}(\Omega)}+C\left\|r_{h}-u_{h}\right\|_{L^{2}(\Omega)}, \\
\left\|A^{-\mu / 2}\left(u-u_{h}\right)_{t}\right\|_{L^{2}(\Omega)} & \leq\left\|A^{-\mu / 2}\left(u-r_{h}\right)_{t}\right\|_{L^{2}(\Omega)}+\left\|A^{-\mu / 2}\left(r_{h}-u_{h}\right)_{t}\right\|_{L^{2}(\Omega)} .
\end{aligned}
$$

Then, (17)-(18) and Lemma 6, together with (54), yield the bound (8).

3. Numerical experiments. We consider the reaction-diffusion system

$$
\left.\begin{array}{rl}
\frac{\partial u_{1}}{\partial t}=1-4 u_{1}+u_{1}^{2} u_{2}+\nu \Delta u_{1} \\
\frac{\partial u_{2}}{\partial t}= & 3 u_{1}-u_{1}^{2} u_{2}+\nu \Delta u_{2}
\end{array}\right\}
$$

known as the Brusselator (see, e.g., [27]), in $\Omega \times[0, T]$, where $\Omega=[0,1]^{2}$ and $T=10$, subject to the Neumann boundary condition

$$
\frac{\partial u_{1}}{\partial n}=\frac{\partial u_{2}}{\partial n}=0 \quad \text { on } \quad \partial \Omega .
$$




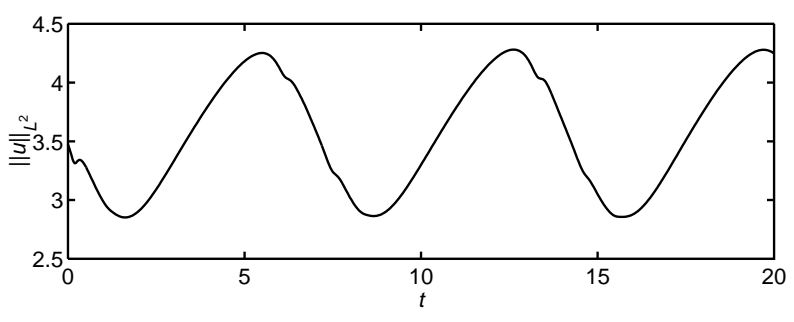

FIG. 1. Evolution of $\|u\|_{L^{2}(\Omega)^{2}}$.

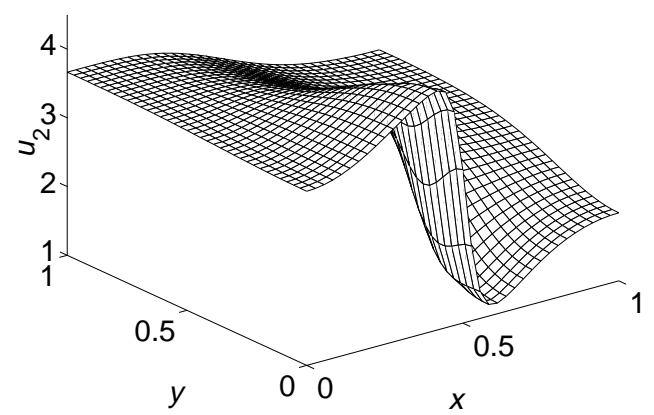

FIG. 2. Component $u_{2}$ of the solution at $T=10$.

Although for simplicity we have developed the analysis for Dirichelet boundary conditions, our analysis can be modified to cover the Neumann boundary conditions case as well.

We set $\nu=0.002$. We complement this system with the initial condition

$$
\begin{aligned}
& u_{1}(x, y, 0)=1-\frac{1}{4}(2 y-1)\left((2 y-1)^{2}-3\right), \\
& u_{2}(x, y, 0)=3-(2 x-1)\left((2 x-1)^{2}-3\right) .
\end{aligned}
$$

The corresponding solution becomes nearly periodic in time, and develops moderately large gradients. Figure 1 shows the evolution of $\|u\|_{L^{2}(\Omega)^{2}}$ up to time $T=20$, and a periodic behavior can be observed. In Figure 2, the component $u_{2}$ of the solution, at time $T=10$, is portrayed; the gradient takes values above 50 in some points of the domain.

In our calculations we take the so-called regular pattern triangulations of $\Omega$, which are induced by the set of nodes $(j / N, k / N), 0 \leq j, k \leq N$, where $N=|\Omega| / h$ is an integer. For the Galerkin method (5) we used Lagrange quadratic elements on these triangulations (i.e., $r=3$ ) and for the postprocessing step, since the solution $u=$ $\left(u_{1}, u_{2}\right)^{T}$ is smooth, we choose Lagrange cubic elements on the same triangulations.

Since the Laplacian is noninvertible when subject to Neumann boundary conditions, for the postprocessing step we solve

$$
\begin{aligned}
\nu \tilde{A}_{h} \tilde{u}_{1, h}+4 \tilde{u}_{1, h} & =1+\tilde{P}_{h}\left(u_{1, h}^{2} u_{2, h}\right)-\frac{d}{d t} u_{1, h}, \\
\nu \tilde{A}_{h} \tilde{u}_{2, h}+\tilde{P}_{h}\left(\tilde{u}_{1, h}^{2} \tilde{u}_{2, h}\right) & =3 \tilde{u}_{1, h}-\frac{d}{d t} u_{2, h},
\end{aligned}
$$

solving first (63) (notice that $\nu \tilde{A}_{h} \tilde{+}_{4} I_{d}$ is invertible) and then (64). 
For the time integration, a variable-formula, variable-coefficient implementation of the backward differentiation formulae (BDF) was used with orders up to six (see, e.g., [26]). This method is widely used in the numerical integration of stiff ordinary differential equations (ODEs), like those arising from the spatial discretizations of dissipative PDEs. This BDF code was previously used in [16],[19], [20]. In [19], this code is shown to be more efficient than other widely used integrators in dissipative PDEs (including semi-implicit methods). Without an efficient time integrator, as shown in [19], [20], it may take an unaffordable amount of computing time to reach the levels of accuracy described below.

For each $h$ used in the triangulations of $\Omega$, every experiment was carried out with different values of the tolerance TOL (an input value to the time integrator) below which local time discretization errors are desired. The smallest error obtained was selected for the plots. There was always a point, depending on $h$, at which further reducing the tolerance did not reduce the errors any more. This means that the error arising from time discretization is much smaller than the error arising from the finite-element spatial discretization. When this happens, one can be sure that the errors observed are due only to the spatial discretization and do not depend on the time integrator used. In view of [19] we were particularly careful to ensure that the dominant error in all the computations presented here was the spatial discretization error in order to avoid wrong conclusions from our numerical experiments. Also, for the computational cost in Figures 4-6 below, the largest tolerance among those with which the spatial discretization error was dominant was used. We refer to this value of the tolerance as the optimal tolerance. A smaller tolerance would result in a larger cost but no smaller error. A larger one, on the other hand, would imply a larger error, but then the same error could be obtained at less cost with the same tolerance and larger $h$.

In Figure 3 we present a convergence diagram showing the errors committed by the methods when used with $h=|\Omega| / N, N=12,24,48$. By errors we mean the difference between the approximations $\left(u_{1, h}, u_{2, h}\right)$ or $\left(\tilde{u}_{1, h}, \tilde{u}_{2, h}\right)$ and the exact solution (at the end of the section we explain how it was obtained). In this and in the rest of the plots, the computational results of the classical quadratic elements are joined by continuous lines, and those of the postprocessed method by discontinuous (dashed) lines. With this, we get an idea of what the result would have been for intermediate values of $h$. It can be seen that as $h$ is decreased from left to right, the errors in both methods decrease from top to bottom, but they decrease faster in the postprocessed method. Measures of the slopes of the plots confirm the rates predicted by the theory (i.e., the errors in the plot decrease like $N^{\text {slope }}=$ const. $h^{- \text {slope }}$ ).

The improved convergence rate of the postprocessed method observed in Figure 3 also implies improved efficiency. This can be checked in Figure 4, where the same errors as in Figure 3 are plotted against the smallest amount of CPU time that the methods needed to achieve them, that is, the cost of integrating the method with the optimal tolerance (notice that, for a given $h$, the optimal tolerance of the postprocessed quadratics is, in general, smaller than that of the quadratics). The reason for this improvement is that the error of the Galerkin method is reduced when the postprocessing is done, but this is done at very little cost (less than $10 \%$ of the whole computation, including the extra cost of integrating in time with smaller tolerances). In fact, the cost of the postprocessing step is equivalent to the cost of roughly 10 time steps, and the number of time steps in the results shown in Figure 4 varies between 109 and 344 . 


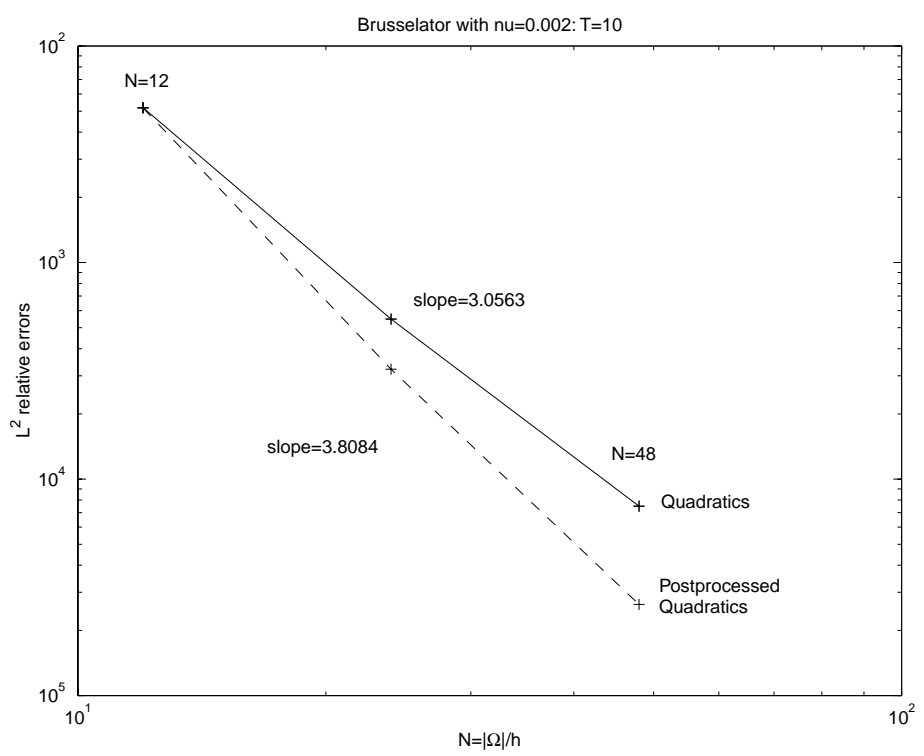

FIG. 3. Convergence diagram.

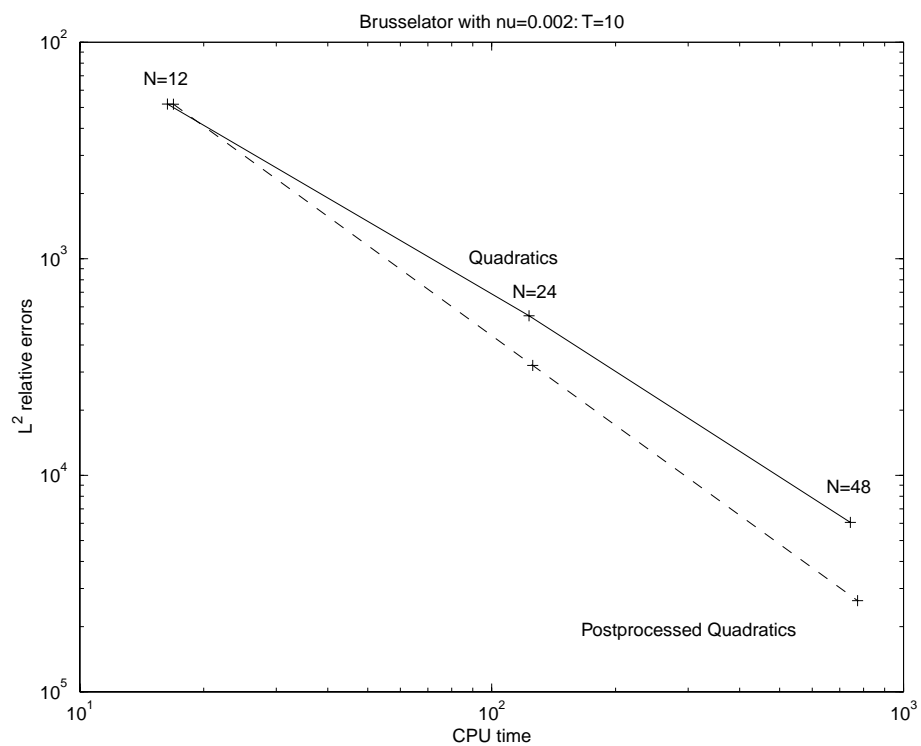

FIG. 4. Efficiency diagram.

The postprocessed quadratic elements are not only more efficient than the classical quadratics but also more efficient than the classical cubic elements, which have the same convergence rate as the postprocessed quadratics. In Figure 5 we have plotted, besides the results of Figure 4, those of the classical cubic elements, that is, the standard Galerkin method (5)-(6) but with $r=4$. These are represented by asterisks joined by dotted lines. Notice how the discontinuous line (postprocessed method) is always on the left (less CPU time) of the dotted line of the cubics. In this example, 


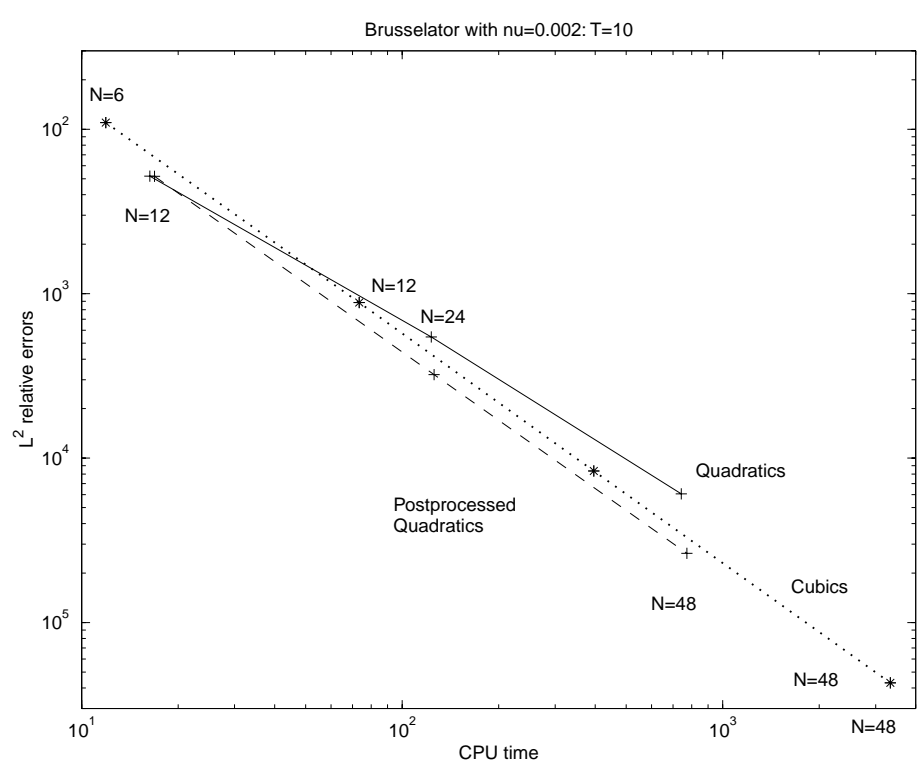

FIG. 5. Efficiency diagram.

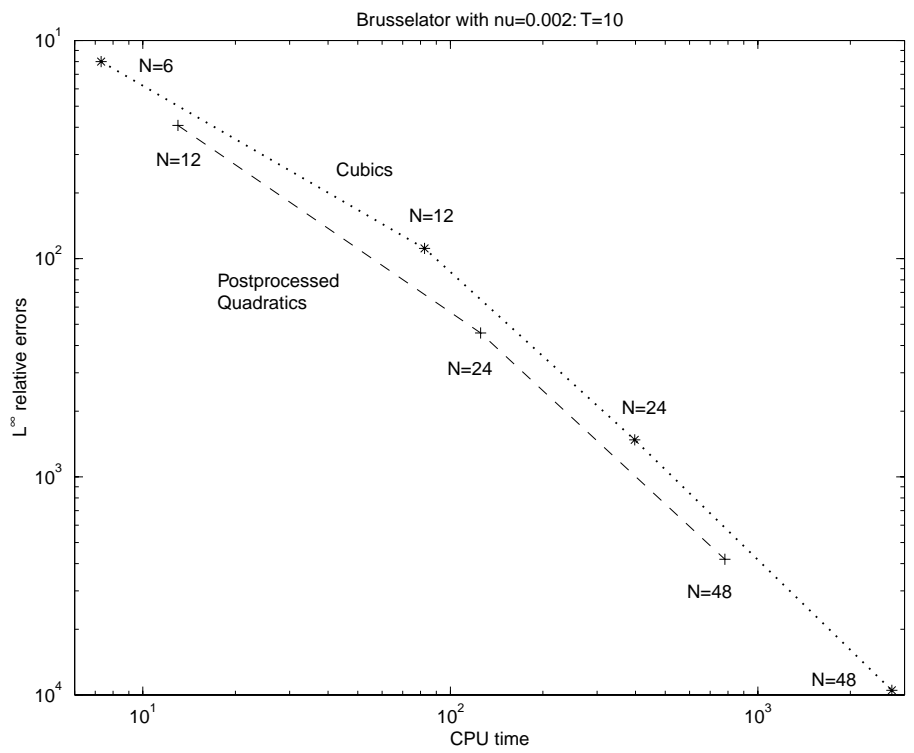

FIG. 6. Efficiency diagram in $L^{\infty}$.

the postprocessed quadratics save between $15 \%$ and $17 \%$ of the cost of the cubics. More significant is the gain in the maximum norm in Figure 6, where the postprocessed quadratics require between $21 \%$ and $25 \%$ less computing time than the classical cubics.

A significant detail can be observed in Figures 5 and 6: The postprocessed method is more efficient than classical methods for quite large errors (already above $10^{-3}$ ). This is in contrast to our previous experience with spectral methods, where, typically, postprocessing brings a substantial improvement only for quite small errors (below $\left.10^{-5}\right)[16],[21]$. 


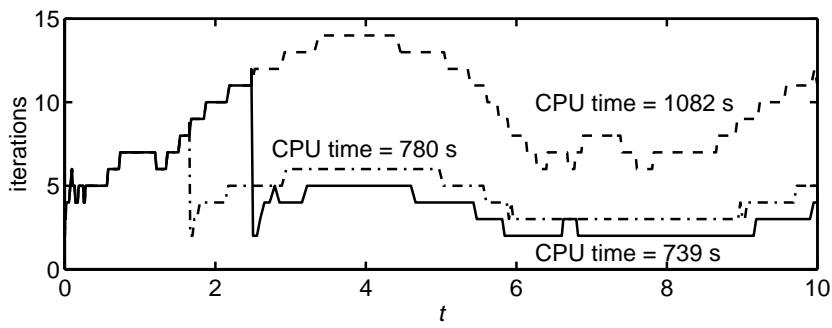

FIG. 7. Evolution of PCG iterations for $h=\sqrt{2} / 48$ and TOL $=0.001:-\cdot-$ MAXIPCG $=8$, MAXIPCG $=11,--$ MAXIPCG $=18818$.

We end this section by commenting on the more relevant details of the methods used. Recall that the time integration is done with the BDF. Since these are fully implicit methods, then, at every time step, a system of nonlinear equations of the form

$$
\alpha v_{h}+(\delta t)\left(\nu A_{h} v_{h}+P_{h} F\left(v_{h}\right)\right)=0
$$

has to be solved, where $\delta t$ is the time steplength. This was done by Newton iteration. The exact Jacobian matrices were replaced by the Jacobian matrix of the linear terms, that is,

$$
\alpha M_{h}+(\delta t) \nu S_{h}
$$

where $M_{h}$ and $S_{h}$ are the mass and stiffness matrices (see, e.g., [45]).

The corresponding linear systems were solved by the preconditioned conjugate gradient method (PCG) with incomplete Cholesky ( $\mathrm{ICH}$ ) factorization of (66) as preconditioner (see, e.g., [23]). Both PCG and ICH algorithms were taken from the SLAP library. A single ICH factorization of (66) proved to be extremely costly (between $5 \%$ to $10 \%$ of the whole computation from $t=0$ to $T=10$ ). Thus, it was computed at the first step and reused in later steps until the PCG did not converge, when it was recalculated again. By convergence in the PCG method we mean achieving a relative error below a prescribed value TOLPCG in less than a fixed number of iterations MAXIPCG.

In our present case, we chose TOLPCG $=10^{-3}$ for the linear systems in the Newton iteration of (65), and $10^{-9}$ for those in the postprocessing (63)-(64). To set the value of MAXIPCG, we performed a practical study for various values of $h$ and settle it to MAXIPCG $=11$, which usually forced the computation of two ICH decompositions. In Figure 7, the number of PCG iterations (the maximum for the two or three linear systems solved per step) for different values of MAXIPCG are shown, together with the CPU times of the corresponding runs. It can be seen how if MAXIPCG is generous (dashed line) the code computes only one ICH factorization at $t=0$ but in the end performs a crippling number of iterations. If MAXIPCG is too tight (dash-dotted line) the second ICH decomposition is computed too soon, at $t=1.6662$; the number of PCG iterations is drastically reduced from then onward, but the overall number of iterations (and hence the cost) is larger than for MAXIPCG $=11$ (continuous line). Notice how in this last case, the number of iterations is drastically reduced at $t=$ 2.5005 when the second ICH factorization is computed, and remains the lowest from then onward. 
For the linear systems in the postprocessing step (63)-(64), it proved far more effective to use the diagonal of the corresponding matrices as a preconditioner. A far greater number of PCG iterations were taken (in the example of Figure 7, 70 for $\tilde{u}_{1, h}$ and 308 for $\tilde{u}_{2, h}$ for systems of 21025 unknowns), but it compensated for the cost of a new ICH factorization (notice that the matrices in the postprocessing step are of larger dimension than those in (66)).

The initial guess for Newton's method in (65) was computed by standard techniques of extrapolation in the BDF code (see, e.g., [26]), and for the PCG method in the corresponding linear systems the initial iterates were set to 0 (notice that the solutions of this systems are the increments of Newton iterations that are supposed to be small). For the linear system in the postprocessing step (63)-(64), the initial guess was the Galerkin approximation $u_{h}$ expressed in the nodal basis of the cubic elements.

It may be asked if other linear algebra techniques would have changed the results shown here. This would be especially the case if a faster (if existing) linear algebra would have favored the cubic elements but not the quadratics (and hence, neither the postprocessed method), although it is difficult to devise why it should be so. Obviously, it is out of the scope of this paper to test all possible linear system solvers, especially when there does not seem to be a clear-cut criterion for when to apply the many existing techniques competing for attention. The PCG used here seems to us a very reasonable choice, especially if we take into account the very low number of iterations that it took to solve the systems in our examples (recall Figure 7). Furthermore, a significant improvement could have been made to the postprocessing step with multilevel techniques (see, e.g., [4]), which would have favored yet further the postprocessed Galerkin method. We tested direct (sparse) solvers, but they were remarkably more costly than the PCG. We also checked that explicit time integrators were much less efficient than the BDF (i.e., the system of ODEs (5) is genuinely stiff).

In all the results shown here, the initial condition (6) was replaced by the interpolant $I_{h}\left(u_{0}\right)$, which is much cheaper to compute than the elliptic projection in (6). We also performed the experiments with (6), and observed that the differences with those shown here were insignificant.

The theoretical ("exact") solution $u$ was computed with cubic elements and $h=\sqrt{2} / 96$, using values of the tolerance ranging from $10^{-9}$ to $10^{-12}$, so that the computed solution taken as exact is reasonably more accurate than those shown in the experiments.

All experiments were carried out on a SUN Ultra-1, Mod. 140 workstation with 64 MB RAM, under Solaris 2.5.1. All programs were written in FORTRAN, used double precision arithmetic, and were compiled with the SparcWorks 4.0 compiler with the -fast option.

\section{4. $L^{\infty}$ analysis.}

4.1. Preliminaries and main result. In comparison to section 2, we further restrict ourselves here in the following sense.

1. The results here apply only to reaction-diffusion equations with nonlinear terms as in (3).

2 . We consider only problems in $\mathbb{R}^{d}$, with $d=2,3$.

(As with the $L^{2}$ analysis, the case $d=1$ is particular and easier to study).

The error estimates in this section depend on

$$
K_{\infty}(u, t)=\|u(\cdot, t)\|_{W^{r, \infty}(\Omega)}+\left\|u_{t}(\cdot, t)\right\|_{W^{r, \infty}(\Omega)}, \quad K_{\infty}(u)=\max _{0 \leq t \leq T} K_{\infty}(u, t) .
$$


Under these conditions, the error bounds of the postprocessed Galerkin method are stated in the following theorem whose proof is given in section 4.3.

THEOREM 3. There exist constants $C=C\left(K_{\infty}(u)\right)>0$ and $h_{0}>0$ such that for every $h \in\left(0, h_{0}\right]$ the solution $\tilde{u}$ of $(7)$ satisfies the following bound:

$$
\begin{aligned}
& \|u-\tilde{u}\|_{L^{\infty}(\Omega)} \leq C h^{r+2-d / 2}|\log (h)|^{4-d / 2} \quad \text { if } r=3 . \\
& \|u-\tilde{u}\|_{L^{\infty}(\Omega)} \leq C h^{r+2}|\log (h)|^{3} \quad \text { if } r \geq 4 .
\end{aligned}
$$

We now state the equivalent to (17)-(18) in the $L^{\infty}$ norm. Instead of (17) we have

$$
\left\|u-r_{h}\right\|_{L^{\infty}(\Omega)}+h\left\|u-r_{h}\right\|_{W^{1, \infty}\left(\Omega \cap \Omega_{h}\right)} \leq C h^{l}\|u\|_{W^{l, \infty}(\Omega)}, \quad 1 \leq l \leq r .
$$

This estimate follows from Theorem 5.1 in [39], (15), and (13). We are unaware of an estimate like (18) in $L^{\infty}$, although similar results in $L^{p}$ can be found in the literature. Thus, we prove the following result.

Lemma 7. Assume that $d \geq 2, r \geq 3$, and $\delta(h)=O\left(h^{\min (r+2,2(r-1))}\right)$. Then there exists a constant $C>0$ such that for $v \in W^{r, \infty}(\Omega)$ the following bound holds:

$$
\left\|A^{-1}\left(v-R_{h} v\right)\right\|_{L^{\infty}(\Omega)} \leq C\|v\|_{W^{r, \infty}(\Omega)} h^{\min (r+2,2(r-1))}|\log (h)| .
$$

Proof. Following [39], we take $\chi_{h}=I_{h}\left(A^{-1}\left(I-R_{h}\right) v\right)$ the interpolant of $A^{-1}(I-$ $\left.R_{h}\right) v$. Then

$$
\left\|A^{-1}\left(I-R_{h}\right) v\right\|_{L^{\infty}(\Omega)} \leq\left\|A^{-1}\left(I-R_{h}\right) v-\chi_{h}\right\|_{L^{\infty}(\Omega)}+\left\|\chi_{h}\right\|_{L^{\infty}(\Omega)} .
$$

Let us take $p$ with $2 \leq p \leq \infty$. For the first term on the right-hand side above we have that (cf. [6, Thm. 3.1.6])

$$
\left\|A^{-1}\left(I-R_{h}\right) v-\chi_{h}\right\|_{L^{\infty}(\Omega)} \leq C h^{2-d / p}\left\|A^{-1}\left(I-R_{h}\right) v\right\|_{W^{2, p}(\Omega)} .
$$

Let us recall the following inequality (see, e.g., [39] and [49]) which, for linear elliptic equations, illustrates the rate at which the Agmon-Douglis-Nirenberg regularity and well-posedness estimate [2] deteriorates as $p \rightarrow \infty$. Namely, there exists a constant $C>0$, which is independent of $p$, such that

$$
\left\|A^{-1} w\right\|_{W^{2, p}(\Omega)} \leq C p\|w\|_{L^{p}(\Omega)}, \quad 2 \leq p<\infty .
$$

Thus, it follows that

$$
\begin{aligned}
\left\|A^{-1}\left(I-R_{h}\right) v-\chi_{h}\right\|_{L^{\infty}(\Omega)} & \leq C p h^{2-d / p}\left\|\left(I-R_{h}\right) v\right\|_{L^{p}(\Omega)} \\
& \leq C p h^{r+2-d / p}\|v\|_{W^{r, p}(\Omega)} .
\end{aligned}
$$

Using (13) we have that $\left\|\chi_{h}\right\|_{L^{\infty}(\Omega)} \leq C h^{-d / p}\left\|\chi_{h}\right\|_{L^{p}(\Omega)}$, and thus

$$
\left\|\chi_{h}\right\|_{L^{\infty}(\Omega)} \leq C h^{-d / p}\left(\left\|\chi_{h}-A^{-1}\left(I-R_{h}\right) v\right\|_{L^{p}(\Omega)}+\left\|A^{-1}\left(I-R_{h}\right) v\right\|_{L^{p}(\Omega)}\right) .
$$

In view of the interpolation error bound (15), (70) and (72) we obtain the following estimate for the first term on the right-hand side of (73):

$$
C h^{-d / p}\left\|\chi_{h}-A^{-1}\left(I-R_{h}\right) v\right\|_{L^{p}(\Omega)} \leq C p h^{r+2-d / p}\|v\|_{W^{r, p}(\Omega)} .
$$

Also, it is well known (see, e.g., [40, Example 3.2]) that

$$
C h^{-d / p}\left\|A^{-1}\left(I-R_{h}\right) v\right\|_{L^{p}(\Omega)} \leq C p h^{\min (r+2,2(r-1))-d / p}\|v\|_{W^{r, p}(\Omega)},
$$

with $C$ independent of $p$ and $h$. Taking $p=|\log (h)|,(71)$ follows easily. 
4.2. $L^{\infty}$ analysis of the Galerkin method. The following resolvent estimate, which is due to Palencia [38], will be needed later for our $L^{\infty}$ error estimates.

Lemma 8. Fix $\alpha \in(0, \pi / 2)$ and $h_{0} \in\left(0, e^{-1}\right]$. Let $\beta(h)$ denote $\beta(h)=\|\left(P_{h}-\right.$ $\left.R_{h}\right) A^{-1} \|_{\infty}\left(=O\left(h^{2}|\log (h)|\right)\right)$. Then there exist positive constants $C_{\infty}$ and $\left.\sigma<1\right)$ such that the bounds

$$
\left\|\left(z I-A_{h}\right)^{-1}\right\|_{\infty} \leq\left\{\begin{array}{cc}
C_{\infty}|z|^{-1} & \text { if }|z| \leq \sigma \beta(h)^{-1} \\
C_{\infty} h^{-d / 2}|z|^{-1} & \text { if }|z| \geq 0
\end{array}\right.
$$

hold for $z \in \mathbb{C}$ with $\pi \geq|\arg (z)| \geq \alpha$ and for $0<h \leq h_{0}$.

Taking for example $a=(d+3) /(2 \sigma \cos (\alpha))$, it easily follows that there exists a constant $C_{0}>0$ such that for $h$ sufficiently small

$$
\left\|e^{-t A_{h}} \chi_{h}\right\|_{L^{\infty}\left(\Omega_{h}\right)} \leq C_{0}\left\|\chi_{h}\right\|_{L^{\infty}\left(\Omega_{h}\right)}, \quad \chi_{h} \in S_{h}, \quad t \geq r_{0}=a \beta(h)|\log (h)| .
$$

We will also use the following bound from Nitsche and Wheeler [36],

$$
\left\|e^{-t A_{h}} \chi_{h}\right\|_{L^{\infty}\left(\Omega_{h}\right)} \leq C_{1}\left\|\chi_{h}\right\|_{L^{\infty}\left(\Omega_{h}\right)}, \quad \chi_{h} \in S_{h}, \quad t \geq 0, \quad r \geq 4 .
$$

The lack of a bound like (76) $\forall t \geq 0$, if $r=3$, is the reason that the convergence rate depends also on the dimension $d$. This will be seen in Lemma 11 below.

Lemma 9. Fix $c_{t h}>0$ and $T>0$. Then there exists a constant $S>0$ such that for $v_{h}:[0, T] \rightarrow S_{h, r}$ satisfying $v_{h}(0)=r_{h}(0)$ and the threshold condition

$$
\left\|r_{h}(t)-v_{h}(t)\right\|_{L^{\infty}\left(\Omega_{h}\right)} \leq c_{t h},
$$

for $t \in\left[0, t_{1}\right]$ where $t_{1} \leq T$, the following bound holds for $h$ sufficiently small:

(78)

$$
\max _{0 \leq t \leq t_{1}}\left\|v_{h}(t)-r_{h}(t)\right\|_{L^{\infty}\left(\Omega_{h}\right)} \leq S \max _{0 \leq t \leq t_{1}}\left\|\int_{0}^{t} e^{-\nu(t-s) A_{h}}\left(\hat{T}_{h}(s)-T_{h}(s)\right) d s .\right\|_{L^{\infty}\left(\Omega_{h}\right)},
$$

where $\hat{T}$ is as in (35).

Proof. We notice that the threshold condition (77) and the fact that $R_{h}$ is bounded ensures that both $\left\|v_{h}\right\|_{L^{\infty}(\Omega)}$ and $\left\|r_{h}\right\|_{L^{\infty}(\Omega)}$ are bounded by some constant $C=$ $C\left(c_{t h}, \max _{0 \leq t \leq T}\|u(t)\|_{L^{\infty}(\Omega)}\right)$. Since $F$ is smooth, it follows that

$$
\left\|F\left(v_{h}\right)-F\left(r_{h}\right)\right\|_{L^{\infty}\left(\Omega_{h}\right)} \leq C\left\|v_{h}-r_{h}\right\|_{L^{\infty}\left(\Omega_{h}\right)} .
$$

Now take norms in (36). For $r \geq 4$, thanks to (76), the fact that $P_{h}$ is bounded in $L^{\infty}$ (recall (20)) and (79) above, standard arguments with the Gronwall lemma lead to $(78)$.

For $r=3$, similar arguments (with a generalized Gronwall lemma as in Lemma 2) will also lead to (78) as long as we prove

$$
\left\|\int_{0}^{t} e^{-\nu(t-s) A_{h}} P_{h}\left(F\left(v_{h}\right)-F\left(r_{h}\right)\right) d s\right\|_{L^{\infty}\left(\Omega_{h}\right)} \leq C \int_{0}^{t} \frac{\left\|v_{h}(s)-r_{h}(s)\right\|_{L^{\infty}\left(\Omega_{h}\right)}}{(t-s)^{\gamma}} d s,
$$

for some $C>0$ and $\gamma<1$. To do this, we consider the value $r_{0}$ in (75) and let us call $\bar{t}=\max \left(0, t-r_{0} / \nu\right)$, so that for $s \in[0, \bar{t}], \nu(t-s) \geq r_{0}$. Then, thanks to (75), (79), and the fact that $P_{h}$ is bounded, we have that

$$
\left\|\int_{\bar{t}}^{t} e^{-\nu(t-s) A_{h}} P_{h}\left(F\left(v_{h}\right)-F\left(r_{h}\right)\right) d s\right\|_{L^{\infty}\left(\Omega_{h}\right)} \leq C \int_{\bar{t}}^{t}\left\|v_{h}(s)-r_{h}(s)\right\|_{L^{\infty}\left(\Omega_{h}\right)} d s .
$$


On the other hand, take $p=d$ and $\kappa=d / 2-1$ so that $1 / p=1 / 2-\kappa / d$. Then, for $s \in[\bar{t}, t]$ and $w_{h} \in S_{h, r}$, thanks to the inverse estimate (13) and Sobolev's lemma, we have that

$$
\begin{aligned}
\left\|e^{-\nu(t-s) A_{h}} w_{h}\right\|_{L^{\infty}\left(\Omega_{h}\right)} & \leq \frac{C}{h}\left\|e^{-\nu(t-s) A_{h}} w_{h}\right\|_{L^{p}\left(\Omega_{h}\right)} \leq \frac{C}{h}\left\|e^{-\nu(t-s) A_{h}} w_{h}\right\|_{H^{\kappa}\left(\Omega_{h}\right)} \\
& \leq \frac{C}{h}\left\|e^{-\nu(t-s) A_{h}} w_{h}\right\|_{L^{2}\left(\Omega_{h}\right)}^{1-\kappa}\left\|A_{h}^{1 / 2} e^{-\nu(t-s) A_{h}} w_{h}\right\|_{L^{2}\left(\Omega_{h}\right)}^{\kappa} \\
& \leq \frac{C}{h(\nu(t-s))^{\kappa / 2}}\left\|w_{h}\right\|_{L^{2}\left(\Omega_{h}\right)} .
\end{aligned}
$$

Now observe that for $s \in[\bar{t}, t]$, since $\nu(t-s) \leq r_{0} \leq C h^{3 / 2}$, it follows that $h^{-1} \leq$ $C\left(\nu(t-s)^{-2 / 3}\right)$. Thus, taking into account that

$$
\frac{\kappa}{2}+\frac{2}{3}=\left(\frac{d}{4}-\frac{1}{2}\right)+\frac{2}{3}=\frac{d}{4}+\frac{1}{6} \leq \frac{3}{4}+\frac{1}{6}=\frac{11}{12}=\gamma<1,
$$

we have that

$$
\left\|e^{-\nu(t-s) A_{h}} w_{h}\right\|_{L^{\infty}\left(\Omega_{h}\right)} \leq \frac{C}{(\nu(t-s))^{\frac{11}{12}}}\left\|w_{h}\right\|_{L^{2}\left(\Omega_{h}\right)} \leq \frac{C\left|\Omega_{h}\right|^{1 / 2}}{(\nu(t-s))^{\frac{11}{12}}}\left\|w_{h}\right\|_{L^{\infty}\left(\Omega_{h}\right)} .
$$

This (replacing $w_{h}$ by $P_{h}\left(F\left(v_{h}\right)-F\left(r_{h}\right)\right)$ ) together with (79) and (81) leads to (80)with $\gamma=11 / 12$.

Having established stability in the previous lemma, we now face the task of estimating the right-hand side of (78) when $v_{h}=u_{h}$. Recall that in the $L^{2}$ case, this was straightforward thanks to the very favorable $L^{2}$-bounds of $A_{h} e^{-\mu(t-s) A_{h}}$, which can be obtained by means of the spectrum of $A_{h}$. In the present case, i.e., the $L^{\infty}$ case, the lack of such bounds for $e^{-\mu(t-s) A_{h}}$ (let alone $A_{h} e^{-\mu(t-s) A_{h}}$ ) makes the task much harder. Since the difficulty lies when $t-s$ is small (recall for example the restriction in (75)), we will decompose the integrals in (78) into two parts corresponding to $t-s$ large (Lemma 10 below) and small (Lemma 11 below).

Lemma 10. Fix $\alpha \in(0, \pi / 2)$, let $\sigma$ and $\beta(h)$ be as in Lemma 8, and set $\tau_{1}=$ $d \beta(h)|\log (h)| /(2 \nu \sigma \cos (\alpha))$. Then there exists a constant $C>0$ such that for $t \geq \tau_{1}$ the following bound holds for $h$ sufficiently small:

$$
\left\|\int_{0}^{t-\tau_{1}} e^{\nu(t-s) A_{h}} T_{h}(s) d s\right\|_{L^{\infty}\left(\Omega_{h}\right)} \leq \frac{C}{\nu}\left|\log \left(\frac{t}{h}\right)\right| \max _{0 \leq s \leq t-\tau_{1}}\left\|A_{h}^{-1} T_{h}(s)\right\|_{L^{\infty}\left(\Omega_{h}\right)} .
$$

Proof. By writing $e^{\nu(t-s) A_{h}} T_{h}(s)=A_{h} e^{\nu(t-s) A_{h}} A_{h}^{-1} T_{h}(s)$, we only need to bound $A_{h} e^{\nu(t-s) A_{h}}$. Let $\Gamma$ be the boundary of the sector $\{z|| \arg (z) \mid \leq \alpha\}$, endowed with the positive orientation. Then

$$
A_{h} e^{-\nu(t-s) A_{h}}=\frac{1}{2 \pi i} \int_{\Gamma} z e^{-\nu(t-s) z}\left(z I-A_{h}\right)^{-1} d z .
$$

We take $\rho_{1}=\sigma \beta(h)^{-1}$, and divide $\Gamma$ in the two parts $\Gamma \cap\left\{|z| \leq \rho_{1}\right\}$ and the two half-lines $\Gamma \cap\left\{|z| \geq \rho_{1}\right\}$. Taking into account the resolvent estimate in Lemma 8, we can write

$$
\left\|A_{h} e^{-\nu(t-s) A_{h}}\right\|_{\infty} \leq 2 C_{\infty} \int_{0}^{\rho_{1}} e^{-\nu(t-s) \cos (\alpha) \rho} d \rho+2 \frac{C_{\infty}}{h^{d / 2}} \int_{\rho_{1}}^{\infty} e^{-\nu(t-s) \cos (\alpha) \rho} d \rho,
$$


so that

$$
\left\|A_{h} e^{-\nu(t-s) A_{h}}\right\|_{\infty} \leq \frac{2 C_{\infty}}{\nu(t-s) \cos (\alpha)}\left(1+\frac{e^{-\nu(t-s) \cos (\alpha) \rho_{1}}}{h^{d / 2}}\right) .
$$

We now notice that for $s \leq t-\tau_{1}$, we have that $t-s \geq \tau_{1}$, and then $-\nu(t-s) \cos (\alpha) \rho_{1} \leq$ $-\nu \tau_{1} \cos (\alpha) \rho_{1}=-d \log (h) / 2=d|\log (h)| / 2$. Thus,

$$
\left\|A_{h} e^{-\nu(t-s) A_{h}}\right\|_{\infty} \leq \frac{4 C_{\infty}}{\nu(t-s) \cos (\alpha)} .
$$

By integrating the right-hand side above with respect to $s$ on the interval $\left[0, t-\tau_{1}\right]$, (83) follows easily.

Lemma 11. Let $\alpha, \sigma, \beta(h)$, and $\tau_{1}$ be as in Lemma 10. Then, there exists a constant $C>0$ such that for $t \geq \tau_{1}$ the following bound holds: for $r=3$,

$$
\left\|\int_{t-\tau_{1}}^{t} e^{\nu(t-s) A_{h}} T_{h}(s) d s\right\|_{L^{\infty}\left(\Omega_{h}\right)} \leq C \log (h)^{3-d / 2} h^{2-d / 2} \max _{t-\tau_{1} \leq s \leq t}\left\|T_{h}(s)\right\|_{L^{\infty}\left(\Omega_{h}\right)},
$$

and for $r \geq 4$,

$$
\left\|\int_{t-\tau_{1}}^{t} e^{\nu(t-s) A_{h}} T_{h}(s) d s\right\|_{L^{\infty}\left(\Omega_{h}\right)} \leq C \log (h)^{2} h^{2} \max _{t-\tau_{1} \leq s \leq t}\left\|T_{h}(s)\right\|_{L^{\infty}\left(\Omega_{h}\right)} .
$$

Proof. The case $r \geq 4$ is a consequence of (76) and the fact that

$$
\tau_{1}=\leq C h^{2} \log (h)^{2} .
$$

For $r=3$, let us take $p=d$ and $\kappa=d / 2-1$, and recall (82). Then

$$
\left\|\int_{t-\tau_{1}}^{t} e^{\nu(t-s) A_{h}} T_{h}(s) d s\right\|_{L^{\infty}\left(\Omega_{h}\right)} \leq \frac{C \tau_{1}^{1-\kappa / 2}}{\nu^{\kappa / 2} h}\left\|T_{h}\right\|_{L^{\infty}\left(\Omega_{h}\right)},
$$

which, since $1-\kappa / 2=3 / 2-d / 4$ and in view of (85), finishes the proof.

Lemma 12. Let $v \in W^{2, \infty}(\Omega)$ and fix $c>0$. Then there exists a constant $C$ that depends on $\|v\|_{W^{2, \infty}(\Omega)}$ and $c$, such that $\forall \chi \in L^{\infty}(\Omega)$ satisfying $\|v-\chi\|_{L^{\infty}(\Omega)} \leq c$, the following bound holds:

$$
\left\|A^{-1}(F(v)-F(\chi))\right\|_{L^{\infty}(\Omega)} \leq C\left(\left\|A^{-1}(v-\chi)\right\|_{L^{\infty}(\Omega)}+\|v-\chi\|_{L^{\infty}(\Omega)}^{2}\right) .
$$

Proof. Since $F$ is smooth, $F^{\prime \prime}$ is bounded in $\left\{x|| x \mid \leq\|v\|_{L^{\infty}(\Omega)}+c\right\}$. Then, using the mean-value theorem, it follows that

$$
\|F(v)-F(\chi)\|_{L^{\infty}(\Omega)} \leq\left\|F^{\prime}(v)(v-\chi)\right\|_{L^{\infty}(\Omega)}+C\|v-\chi\|_{L^{\infty}(\Omega)}^{2} .
$$

Since $\left\|A^{-1}\right\|_{\infty}<+\infty$, we need only to bound the $\left\|A^{-1} F^{\prime}(v)(v-\chi)\right\|_{L^{\infty}(\Omega)}$. Let us denote $\epsilon=v-\chi$ and $\mathcal{E}=A^{-1} \epsilon$. Since $\Delta\left(F^{\prime}(v) \mathcal{E}\right)=\mathcal{E} \Delta F^{\prime}(v)+2 \nabla F(v) \cdot \nabla \mathcal{E}+F^{\prime}(v) \epsilon$, we have that

$$
A^{-1} F^{\prime}(v) \epsilon=F^{\prime}(v) \mathcal{E}-A^{-1}\left(\mathcal{E} \Delta F^{\prime}(v)\right)-2 A^{-1}\left(\nabla F^{\prime}(v) \cdot \nabla \mathcal{E}\right) .
$$


It is clear that the first two terms on the right-hand side above are bounded by the right-hand side of (86), so we need only to bound the third term. Take $p=d+1$; then

$$
\left\|A^{-1}\left(\nabla F^{\prime}(v) \cdot \nabla \mathcal{E}\right)\right\|_{L^{\infty}(\Omega)} \leq C\left\|\nabla A^{-1}\left(\nabla F^{\prime}(v) \cdot \nabla \mathcal{E}\right)\right\|_{L^{p}(\Omega)} .
$$

We estimate the right-hand side above by duality. Take $\phi \in C_{0}^{\infty}(\Omega)^{2}$. Then

$$
\begin{aligned}
\left|\left(\nabla A^{-1}\left(\nabla F^{\prime}(v) \cdot \nabla \mathcal{E}\right), \phi\right)\right| & =\left|\left(A^{-1}\left(\nabla F^{\prime}(v) \cdot \nabla \mathcal{E}\right), \operatorname{div}(\phi)\right)\right| \\
& \left.=\mid\left(\nabla F^{\prime}(v) \cdot \nabla \mathcal{E}\right), A^{-1} \operatorname{div}(\phi)\right) \mid \\
& =\mid\left(\mathcal{E}, \operatorname{div}\left(A^{-1} \operatorname{div}(\phi) \nabla F^{\prime}(v)\right) \mid\right. \\
& \leq\|\mathcal{E}\|_{L^{p}(\Omega)} \| \operatorname{div}\left(A^{-1} \operatorname{div}(\phi) \nabla F^{\prime}(v) \|_{L^{q}(\Omega)}\right. \\
& \leq C\|\mathcal{E}\|_{L^{\infty}(\Omega)}\|v\|_{W^{2, \infty}(\Omega)}\left\|A^{-1} \operatorname{div}(\phi)\right\|_{W^{1, q}(\Omega)} \\
& \leq C\|\mathcal{E}\|_{L^{\infty}(\Omega)}\|v\|_{W^{2, \infty}(\Omega)}\|\operatorname{div}(\phi)\|_{W^{-1, q}(\Omega)} \\
& \leq C\|\mathcal{E}\|_{L^{\infty}(\Omega)}\|v\|_{W^{2, \infty}(\Omega)}\|\phi\|_{L^{q}(\Omega)} \cdot \quad \square
\end{aligned}
$$

Lemma 13. There exists a constant $C$ that depends on $K_{\infty}(u)$, which is given in (67), such that for $t \in[0, T]$ the following bounds hold:

$$
\begin{aligned}
\left\|T_{h}(t)\right\|_{L^{\infty}\left(\Omega_{h}\right)} & \leq C h^{r}, \\
\left\|A_{h}^{-1} T_{h}(t)\right\|_{L^{\infty}\left(\Omega_{h}\right)} & \leq C h^{r+\bar{\mu}}|\log (h)|, \\
\left\|A^{-1} T_{h}(t)\right\|_{L^{\infty}\left(\Omega_{h}\right)} & \leq C h^{r+\bar{\mu}}|\log (h)|,
\end{aligned}
$$

where $\bar{\mu}=2$ if $r \geq 4$ and $\bar{\mu}=1$ otherwise.

Proof. The bound (87) is a direct consequence of the mean-value theorem, (70) and the fact that $\left\|P_{h}\right\|_{\infty}$ is finite. To prove (88) we notice that $A_{h}^{-1} P_{h}=R_{h} A^{-1}$. Since $\left\|R_{h}\right\|_{\infty}$ is finite (recall (21)) we have that

$$
\left\|A_{h}^{-1} T_{h}\right\|_{L^{\infty}(\Omega)} \leq C\left(\| A^{-1}\left(F\left(r_{h}\right)-F(u)\left\|_{L^{\infty}(\Omega)}+\right\| A^{-1}\left(r_{h}-u\right)_{t} \|_{L^{\infty}(\Omega)}\right) .\right.
$$

Then (88) follows from Lemma 12 and (71). Finally, for (89), we write $A^{-1}=\left(A^{-1}-\right.$ $\left.A_{h}^{-1} P_{h}\right)+A_{h}^{-1} P_{h}$. Since from (70) and (74) we have that $\left\|A^{-1}-A_{h}^{-1} P_{h}\right\|_{\infty}=\|(I-$ $\left.R_{h}\right) A^{-1} \|_{\infty}=O\left(h^{2}|\log (h)|\right)$, then (89) follows from (87) and (88).

THEOREM 4 . There exist a constant $C$ that depends on $K_{\infty}(u)$, which is given in (67), and a constant $h_{0}>0$ such that for $0<h \leq h_{0}$ the following bound holds:

$$
\max _{0 \leq t \leq T}\left\|r_{h}(t)-u_{h}(t)\right\|_{L^{\infty}(\Omega)} \leq C h^{r+\tilde{\mu}}|\log (h)|^{\tilde{r}},
$$

where $\tilde{\mu}=2$ when $r \geq 4$ (otherwise $\tilde{\mu}=2-d / 2$ ) and $\tilde{r}=2$ when $r \geq 4$ (otherwise $\tilde{r}=3-d / 2)$.

Proof. The proof is a direct consequence of Lemmas 9,10,11, and 13. One has only to check that the threshold condition (77) holds, but this, as in Theorem 2, is easily proven by standard continuity arguments.

4.3. Analysis of the postprocessing step in $\boldsymbol{L}^{\infty}$. In this section, as in section 2.3, we first prove Theorem 3. Then we provide an approximation $\tilde{u}_{h}$, in an appropriate finite-element space, to $\tilde{u}$ such that $\left\|\tilde{u}-\tilde{u}_{h}\right\|_{L^{\infty}(\Omega)}$ is of the same order as $\|\tilde{u}-u\|_{L^{\infty}(\Omega)}$. 
Proof of Theorem 3. One has only to follow the reasoning of section 2.3, replacing $L^{2}(\Omega)$ by $L^{\infty}(\Omega)$, and concentrating on the case $\mu=2$. Taking the $L^{\infty}$ norms in (59) and applying Lemma 12 we obtain that

$$
\|u-\tilde{u}\|_{L^{\infty}(\Omega)} \leq C\left(\left\|A^{-1}\left(u-u_{h}\right)\right\|_{L^{\infty}(\Omega)}+\left\|u-u_{h}\right\|_{L^{\infty}(\Omega)}^{2}\right)+\left\|A^{-1}\left(u-u_{h}\right)_{t}\right\|_{L^{\infty}(\Omega)} .
$$

We write $u-u_{h}=\left(u-r_{h}\right)+\left(r_{h}-u_{h}\right)$. Then, in view of (70) and the estimation of $\left\|r_{h}-u_{h}\right\|_{L^{\infty}(\Omega)}$ in Theorem 4 we have that

$$
\begin{aligned}
\|u-\tilde{u}\|_{L^{\infty}(\Omega)} \leq & C\left\|A^{-1}\left(u-r_{h}\right)\right\|_{L^{\infty}(\Omega)}+\left\|A^{-1}\left(u-r_{h}\right)_{t}\right\|_{L^{\infty}(\Omega)} \\
& +\left\|A^{-1}\left(r_{h}-u_{h}\right)_{t}\right\|_{L^{\infty}(\Omega)}+ \begin{cases}C h^{r+2-r / d}|\log (h)|^{3-d / 2}, & r=3, \\
C h^{r+2}|\log (h)|^{2}, & r \geq 4 .\end{cases}
\end{aligned}
$$

Lemma 7 shows that the first two terms on the right-hand side above are bounded by the right-hand sides of (68)-(69). In order to estimate $\left\|A^{-1}\left(r_{h}-u_{h}\right)_{t}\right\|_{L^{\infty}(\Omega)}$, we follow the proof in Lemma 6 . Thus we return to (57). We have already estimated $\left\|A^{-1} T_{h}\right\|_{L^{\infty}(\Omega)}$ in Lemma 13. The term $A^{-1} A_{h}\left(u_{h}-r_{h}\right)$ is treated as in Lemma 6 , but now notice that $\left\|\left(A^{-1}-A_{h}^{-1}\right) P_{h}\right\|_{\infty}=O\left(h^{2}|\log (h)|\right)$, which accounts for the extra $|\log (h)|$ term in (68)-(69) with respect to the bound (90) of $u-r_{h}$ in Theorem 4. The second term on the right-hand side of (57) can be bounded as follows. Notice that $\left\|A^{-1} P_{h}\right\|_{\infty}<+\infty$, so that in view of (79) and Theorem 4.2, we have that

$$
\begin{aligned}
\left\|A^{-1} P_{h}\left(F\left(u_{h}\right)-F\left(r_{h}\right)\right)\right\|_{L^{\infty}(\Omega)} & \leq C\left\|F\left(u_{h}\right)-F\left(r_{h}\right)\right\|_{L^{\infty}(\Omega)} \\
& \leq C\left\|u_{h}-r_{h}\right\|_{L^{\infty}(\Omega)} \leq C h^{r+\tilde{\mu}}|\log (h)|^{\tilde{r}} .
\end{aligned}
$$

Let us now consider the solution $\tilde{u}_{h}=\tilde{R}_{h} \tilde{u}$ of (56), where the finite-element space $\tilde{S}_{h}$ is as in section 2.3 , but adequately modified to account for the difference in convergence rates in $L^{2}$ and $L^{\infty}$. Since $\tilde{R}_{h}$ is bounded in $L^{\infty}$ we have that $\forall \tilde{\chi}_{h} \in \tilde{S}_{h}$

$$
\begin{aligned}
\left\|\tilde{u}-\tilde{u}_{h}\right\|_{L^{\infty}(\Omega)}=\left\|\left(I-\tilde{R}_{h}\right) \tilde{u}\right\|_{L^{\infty}(\Omega)} & =\left\|\left(I-\tilde{R}_{h}\right)\left(\tilde{u}-\tilde{\chi}_{h}\right)\right\|_{L^{\infty}(\Omega)} \\
& \leq C\left\|\tilde{u}-\tilde{\chi}_{h}\right\|_{L^{\infty}(\Omega)} .
\end{aligned}
$$

As in section 2.3, replacing $\tilde{\chi}_{h}$ by the interpolant $\tilde{I}_{h}(u)$ of $u$ in $\tilde{S}_{h}$ and writing $\tilde{u}-$ $\tilde{I}_{h}(u)=(\tilde{u}-u)+\left(u-\tilde{I}_{h}(u)\right)$, then with Theorem 3 and the interpolation error $(15)$ we conclude that $\left\|\tilde{u}-\tilde{u}_{h}\right\|_{L^{\infty}(\Omega)}$ is of the same order as $\|\tilde{u}-u\|_{L^{\infty}(\Omega)}$.

Acknowledgments. The authors thank Professor A. H. Schatz for his valuable suggestions and Professor C. Palencia for helpful discussions.

\section{REFERENCES}

[1] R. A. Adams, Sobolev Spaces, Academic Press, New York, 1975.

[2] S. Agmon, A. Douglis And L. Nirenberg, Estimates near the boundary for solutions of elliptic partial differential equations I and II, Comm. Pure Appl. Math., 12 (1959), pp. 623-727; 17 (1964), pp. 35-92.

[3] A. Ait Ou Ammi and M. Marion, Nonlinear Galerkin methods and mixed finite elements: Two-grid algorithms for the Navier-Stokes equations, Numer. Math., 68 (1994), pp. 189213.

[4] R. E. BAnK, Hierarchical bases and the finite element method, in Acta Numerica 5, A. Iserles, ed., Cambridge University Press, Cambridge, 1996, pp. 1-43.

[5] R. E. BANK AND D. J. Rose, Analysis of multilevel iterative methods for nonlinear finite element equations, Math. Comp., 39 (1982), pp. 453-465. 
[6] P. G. Ciarlet, The Finite Element Method for Elliptic Problems, North-Holland, Amsterdam, 1978.

[7] P. Constantin and C. Foias, Navier-Stokes Equations, Chicago Lectures in Math., The University of Chicago, Chicago, 1988.

[8] P. Constantin, C. Foias, B. Nicolenko, and R. Temam, Integral and Inertial Manifolds for Dissipative Partial Differential Equations, Springer-Verlag, New York, 1988.

[9] C. N. DaWson And M. F. Wheeler, Two-grid methods for mixed finite element approximations of nonlinear parabolic problems, Contemp. Math. 180, AMS, Providence, RI, 1994, pp. 192204.

[10] A. Debussche And M. Marion, On the construction of families of approximate inertial manifolds, J. Differential Equations, 100 (1992), pp. 173-201.

[11] C. Devulder And M. Marion, A class of numerical algorithms for large time integration: The nonlinear Galerkin method, SIAM J. Numer. Anal., 29 (1992), pp. 462-483.

[12] C. Foias, M. S. Jolly, I. G. Kevrekidis, G. R. Sell, and E. S. Titi, On the computation of inertial manifolds, Phys. Lett. A, 131 (1988), pp. 433-436.

[13] C. Foias, O. Manley, And R. Temam, Modelling of the interaction of small and large eddies in two dimensional turbulent flows, RAIRO Modél. Math. Anal. Numér., 22 (1988), pp. 93114.

[14] C. Foias, G. R. Sell, and R. Temam, Inertial manifolds for nonlinear evolutionary equations, J. Differential Equations, 73 (1988), pp. 309-353.

[15] C. Foias, G. R. Sell, and E. S. Titi, Exponential tracking and approximation of inertial manifolds for dissipative nonlinear equations, J. Dynam. Differential Equations, 1 (1989), pp. 199-243.

[16] J. de Frutos, B. García-Archilla, and J. Novo, A postprocessed Galerkin method with Chebyshev and Legendre polynomials, Numer. Math., to appear.

[17] J. De Frutos And J. Novo, A Spectral Element Method for the Navier-Stokes Equations with Improved Accuracy, manuscript.

[18] J. De Frutos and J. Novo, A postprocess based improvement of the spectral element method, Appl. Numer. Math., to appear.

[19] B. García-Archilla, Some practical experience with the time integration of dissipative equations, J. Comput. Phys., 122 (1995), pp. 25-29.

[20] B. García-Archilla and J. De Frutos, Time integration of the nonlinear Galerkin method, IMA J. Numer. Anal., 15 (1995), pp. 221-244.

[21] B. García-Archilla, J. Novo, and E. S. Titi, Postprocessing the Galerkin method: A novel approach to approximate inertial manifolds, SIAM J. Numer. Anal., 35 (1998), pp. 941-972.

[22] B. García-Archilla, J. Novo, and E. S. Titi, An approximate inertial manifold approach to postprocessing Galerkin methods for the Navier-Stokes equations, Math. Comp., 68 (1999), pp. 843-911.

[23] G. H. Golub and C. F. van Loan, Matrix Computations, 2nd ed., The John Hopkins University Press, Baltimore, 1989

[24] J. HaLE, Asymptotic Behavior of Dissipative Systems, Math. Surveys Monogr. 25, AMS, Providence, RI, 1988.

[25] D. Henry, Geometric Theory of Semilinear Parabolic Equations, Springer-Verlag, Berlin, 1988.

[26] E. Hairer, H. P. Nørsett, and G. Wanner, Solving Ordinary Differential Equations I, 2nd ed., Springer-Verlag, Berlin, 1993.

[27] E. Hairer and G. Wanner, Solving Ordinary Differential Equations II, 2nd ed., SpringerVerlag, Berlin, 1996.

[28] M. S. Jolly, I. G. Kevrekidis, and E. S. Titi, Approximate inertial manifolds for the Kuramoto-Sivashinsky equation: Analysis and computations, Phys. D, 44 (1990), pp. 3860.

[29] D. A. Jones AND E. S. Titi, $C^{1}$ approximating inertial manifolds for dissipative nonlinear equations, J. Differential Equations, 127 (1996), pp. 54-86.

[30] C. R. Laing, A. McRobie, and J. M. T. Thompson, The post-processed Galerkin method applied to nonlinear shell vibrations, Dynam. Stability Systems., 14 (1999), pp. 166-181.

[31] L. MANSFIELD, On the solution of nonlinear finite element systems, SIAM J. Numer. Anal., 17 (1998), pp. 752-765.

[32] M. Marion and R. Temam, Nonlinear Galerkin methods, SIAM J. Numer. Anal., 26 (1989), pp. 1139-1157.

[33] M. Marion and R. Temam, Nonlinear Galerkin methods: The finite element case, Numer. Math., 57 (1990), pp. 1-22.

[34] M. MARION AND J. XU, Error estimates on a new nonlinear Galerkin method based on two-grid finite elements, SIAM J. Numer. Anal., 32 (1995), pp. 1170-1184. 
[35] G. Nabh And R. RANnACher, A comparative study of nonlinear Galerkin finite element methods for one-dimensional evolution problems, East-West J. Numer. Math., 5 (1997), pp. 67144.

[36] J. A. Nitsche And M. F. Wheeler, $L_{\infty}$ boundedness for the finite element Galerkin method for parabolic problems, Numer. Funct. Anal., 11 (1981), pp. 325-353.

[37] J. Novo, E. S. TITI, AND S. WYNNE, Efficient methods using high accuracy approximate inertial manifolds, Numer. Math., to appear.

[38] C. Palencia, Maximum norm analysis of completely discrete finite element methods for parabolic problems, SIAM J. Numer. Anal., 33 (1996), pp. 1654-1668.

[39] A. H. Schatz And L. B. Wahlbin, On the quasi-optimality in $L^{\infty}$ of the $\dot{H}_{1}$-projection into finite-element spaces, Math. Comp., 38 (1982), pp. 1-21.

[40] A. H. Schatz, I. H. Sloan, AND L. B. WahlBin, Superconvergence in finite element methods and meshes that are locally symmetric with respect to a point, SIAM J. Numer. Anal., 33 (1996), pp. 505-521.

[41] R. Temam, Infinite Dimensional Dynamical Systems in Mechanics and Physics, Appl. Math. Sci. 68, Springer-Verlag, Berlin, 1988.

[42] R. Temam, Attractors for the Navier-Stokes equations: Localization and approximation, J. Fac. Sci. Univ. Tokyo IA Math., 36 (1989), pp. 629-647.

[43] E. S. Tiтi, On approximate inertial manifolds to the Navier-Stokes equations, J. Math. Anal. Appl., 149 (1990), pp. 540-557.

[44] L. B. WAhlBin, Superconvergence in Galerkin Finite Element Methods, Lecture Notes in Math. 1605, Springer-Verlag, Berlin, 1995.

[45] R. Wait And A. R. Mitchell, Finite Element Analysis and Applications, John Wiley \& Sons, Chichester, 1995.

[46] R. Wallace And D. Sloan, Numerical solutions of a nonlinear dissipative system using a pseudospectral method and inertial manifolds, SIAM J. Sci. Comput., 16 (1995), pp. 10491070.

[47] J. XU, A novel two-grid method for semilinear elliptic equations, SIAM J. Sci. Comput., 15 (1994), pp. 231-237.

[48] J. XU, Two-grid discretization techniques for linear and nonlinear problems, SIAM J. Numer. Anal., 33 (1996), pp. 1759-1777.

[49] V. I. Yudovitch, Nonstationary flow of an ideal incompressible liquid, Zh. Vychisl. Mat. i. Mat. Fiz., 6 (1963), pp. 1032-1066. 